\title{
A network-based approach reveals the dysregulated transcriptional regulation in non- alcohol fatty liver disease
}

Hong Yang ${ }^{1}$, Muhammad Arif ${ }^{1}$, Meng Yuan ${ }^{1}$, Xiangyu Li ${ }^{1}$, Ko Eun Shong ${ }^{1}$, Hasan Turkez ${ }^{2}$, Jens Nielsen ${ }^{3.4}$, Mathias Uhlén ${ }^{1}$, Jan Borén ${ }^{5}$, Cheng, Zhang ${ }^{1,6, ~ *}$, Adil Mardinoglu 1,7, *

${ }^{1}$ Science for Life Laboratory, KTH - Royal Institute of Technology, Stockholm, Sweden;

${ }^{2}$ Department of Medical Biology, Faculty of Medicine, Atatürk University, Erzurum, Turkey;

${ }^{3}$ Department of Biology and Biological Engineering, Chalmers University of Technology, Gothenburg SE-41296, Sweden;

${ }^{4}$ BioInnovation Institute, Copenhagen N DK-2200, Denmark;

${ }^{5}$ Department of Molecular and Clinical Medicine, University of Gothenburg and Sahlgrenska University Hospital, Gothenburg, Sweden;

${ }^{6}$ School of Pharmaceutical Sciences, Zhengzhou University, Zhengzhou, PR China;

${ }^{7}$ Centre for Host-Microbiome Interactions, Faculty of Dentistry, Oral \& Craniofacial Sciences, King's College London, London, United Kingdom;

*Correspondence: Dr. Cheng Zhang (cheng.zhang@scilifelab.se), Dr. Adil Mardinoglu (adilm@scilifelab.se).

Keywords: NAFLD; metabolism; transcriptional regulation; network analysis. 


\begin{abstract}
Non-alcohol-related fatty liver disease (NAFLD) is a leading cause of chronic liver disease worldwide. We performed network analysis to investigate the dysregulated biological processes in the disease progression and revealed the molecular mechanism underlying NAFLD. Based on network analysis, we identified a highly conserved disease-associated gene module across three different NAFLD cohorts and highlighted the predominant role of key transcriptional regulators associated with lipid and cholesterol metabolism. In addition, we revealed the detailed metabolic differences between heterogenous NAFLD patients through integrative systems analysis of transcriptomic data and liver-specific genome-scale metabolic model. Furthermore, we identified transcription factors (TFs), including SREBF2, HNF4A, SREBF1, YY1 and KLF13, showing regulation of hepatic expression of genes in the NAFLDassociated modules and validated the TFs using data generated from a mouse NAFLD model. In conclusion, our integrative analysis facilitated our understanding of the regulatory mechanism of these perturbed TFs and associated biological processes.
\end{abstract}




\section{INTRODUCTION}

Non-alcohol fatty liver disease (NAFLD) is considered as one of the most important causes of liver disease, worldwide (Asrani et al., 2019). The global prevalence of NAFLD was estimated to be $25 \%$ and has increased rapidly (Huang et al., 2021; Younossi et al., 2018; Younossi et al., 2016). NAFLD is characterized by the hepatic accumulation of triglycerides, spanning from simple non-alcohol fatty liver (NAFL) to non-alcohol steatohepatitis (NASH) that might progress to cirrhosis and hepatocellular carcinoma (HCC) (Friedman et al., 2018; Huang et al., 2021; Ioannou et al., 2019). Moreover, NAFLD is strongly associated with obesity, diabetes and cardiovascular disease; therefore, it drastically increased in these patient groups (Golabi et al., 2019; Ye et al., 2020; Younossi et al., 2019). However, no effective therapies are yet approved for NAFLD, even though extensive research activities have been carried out (ElAgroudy et al., 2019; Mullard, 2020; Newsome et al., 2021; Stower, 2021). Hence, a comprehensive understanding of the underlying molecular mechanism of NAFLD is critical for the development of novel approaches for its prevention and treatment.

Biological networks provide a robust framework for integrating omics data, elucidating pathophysiological responses and revealing the underlying molecular mechanisms involved in the progression of disease (Calabrese et al., 2017; Mardinoglu et al., 2018; Nayak et al., 2009). Biological networks, including protein-protein interaction networks (PPINs), transcriptional regulatory networks (RNs), gene co-expression networks (GCNs), genome-scale metabolic models (GEMs) and integrated networks (INs), are widely used in systems analysis (Mardinoglu et al., 2018). The central goal of biological network analysis is to identify critical functional units (so-called modules) and their constituent genes (Califano et al., 2012; Choobdar et al., 2019). Such functional modules often linked to disease processes, in which key drivers are highly enriched and provide insights into the disease pathogenesis (Cerami et al., 2010; Huan et al., 2013; Wainberg et al., 2021). In particular, GCNs for 17 human cancers and 46 human tissues have been generated and used to gain insights into disease mechanisms by identifying the key biological components of the cancers or tissues (Arif et al., 2021; Lee et 
al., 2018; Uhlen et al., 2017). GEMs, reconstructed by incorporating all biochemical reactions and transport processes in a cell or tissue, have been extensively used to discover potential biomarkers and drug targets, as well as to reveal the mode of action of a drug (Lewis and Kemp, 2021; Mardinoglu et al., 2018).

To date, GCNs have been used for investigating the causal mechanisms underlying NAFLD using mouse population data (Chella Krishnan et al., 2018) and human population data (Zhang et al., 2020) and for integrative analysis of mouse model data and patients data (Saeed, 2021). However, there is still a lack of holistic studies in which adequately samples covering a large spectrum of disease severity were analysed. Moreover, the heterogeneity of clinical manifestations among NAFLD patients acts as an essential impediment for the discovery of critical pathogenic drivers, and thus systematic analysis is needed (Alonso et al., 2017). Recently, several liver-biopsy proved transcriptomics data from large patients' cohorts had been conducted (Azzu et al., 2021; Govaere et al., 2020; Hoang et al., 2019), and these datasets may be used to provide significant functional insights based on network analysis that cannot be derived from individual gene-level analysis.

In this study, we employed an integrative systems biology approach by integrating NAFLD transcriptomics data with biological networks and elucidated the molecular mechanisms underlying NAFLD progression. We first generated GCNs for liver tissue of normal and NAFLD patients based on transcriptomics data and identified the perturbated modules associated with the severity of NAFLD. Second, we employed a liver-specific GEM called iHepatocytes 2322 to analyse the differential expression data, and gained insights into detailed metabolic differences in NAFLD. Third, we subsequently validated the perturbated modules using transcriptomics data from another two independent studies and highlighted the diseaseassociated modules that are conserved across multiple NAFLD cohorts by combing functional and topological similarities. Next, we used a liver cancer dataset in the cancer genome atlas (TCGA) to investigate if the dysregulated expression of genes in the disease-associated modules is relevant to patient outcome. Finally, we performed transcription factor (TF)-target 
regulatory network analysis, identified TFs that regulate disease-associated modules and validated those TFs using the transcriptomics data from a mouse NAFLD model fed by high sucrose diet (HSD).

\section{RESULTS}

\section{Generation of co-expression networks for liver tissue}

We identified the robust co-expressed genes showing transcriptional differences between the liver tissue of normal subjects and NAFLD patients. We first constructed GCNs of liver tissue based on the transcriptomics data from the Genotype-Tissue Expression (GTEx) database (GTEx analysis V8) (Consortium, 2013) and NAFLD cohort including 10 normal samples, 50 patients with NAFL and 155 patients with NASH (Govaere et al., 2020) (Figure 1A). We filtered out lowly-expressed genes for each dataset based on their mean gene expression level (TPMs <1) and performed Spearman's rank correlation test between each gene pair. All $\mathrm{p}$ values were adjusted by FDR correction (Benjamini-Hochberg). Afterwards, we remained gene pairs with significantly positive correlation (coefficient $>0$ with FDR $<0.05$ ) on the networks (Figure 1A) and used the Leiden algorithm (Traag et al., 2019) to identify modules of genes from the network. In total, Leiden graph-based clustering identified six and five modules of genes in the GTEx cohort and NAFLD cohort, respectively. Each module in the same cohort consists of uniquely assigned genes with a substantial similarity between gene expression (Figure 1B; Dataset S1). Of note, we found that gene members of any module in the GTEx cohort were different from that of modules in the NAFLD cohort even though $95.9 \%$ genes comprising modules in the NAFLD cohort were included by GTEx modules (Figure 1C).

\section{Identification of perturbated modules in NAFLD}

We investigated whether the differences in module composition correlated with the molecular changes underlying NAFLD progression. We first identified differentially expressed genes (DEGs) to reveal the global transcriptomic differences in the liver of patients with NAFLD. We observed that 4,051 and 4,281 genes were significantly upregulated (adjusted p-value $<$ 
0.05) between NAFL and control samples and between NASH and normal samples, respectively (Figure 1D; Dataset S2). Enrichment analysis in KEGG pathway showed that the upregulated DEGs are mostly enriched in the pathways associated with endocytosis, axon guidance, adherens junction, insulin resistance and insulin signalling (Figure 1E; Dataset S3). Moreover, we found that 3,936 and 3,911 genes were significantly downregulated between NAFL and control samples and between NASH and normal samples, respectively (Figure 1D; Dataset S2). Enrichment analysis showed that downregulated DEGs enriched in pathways associated with oxidative phosphorylation, spliceosome, thermogenesis, and proteasome (Figure 1E).

We also examined the enrichment of those dysregulated DEGs associated with NAFLD in each co-expression module identified in GTEx and NAFLD cohort data. The results showed that module 1 and module 3 of the NAFLD cohort with 215 samples (cohort2215_M1 and cohort_215_M3) are significantly enriched (hypergeometric test p-value $\approx 0$ ) by upregulated and downregulated DEGs associated with NAFLD, respectively (Figure 1F). In particular, 66.6\% of genes $(1,431$ out of 2,149) in cohort_215_M1 and 94.6\% of genes (314 out of 332) in cohort_215_M3 are significantly upregulated and downregulated in NAFL vs control groups, respectively. $65.8 \%$ of genes in cohort1_215_M1 and 94\% of genes (312 out of 332) in cohort_215_M3 are significantly upregulated and downregulated in NASH vs control groups, respectively. Notably, we found both cohort_215_M1 and cohort_215_M3 are significantly overlapped (hypergeometric test $\mathrm{p}$-value $=2.17 \times 10^{-14}$ and $3.11 \times 10^{-8}$ ) with module 1 in GTEx cohort (GTEx_M1), which were overrepresented by both upregulated and downregulated DEGs associated with NAFLD (Figure 1F). Interestingly, KEGG enrichment analysis of genes in those modules suggests that the significantly enriched pathways are consistent with the dysregulated pathways enriched by DEGs (Figure 1E; Figure S2A). Moreover, we found cohort_215_M4 are significantly enriched by upregulated genes (19 and 25 out of 38 in NAFL vs control and NASH vs control, respectively) and only significantly overlapped with GTEx_M3 (hypergeometric test $\mathrm{p}$-value $=4.11 \times 10^{-13}$ ). Taken together, co-expression network 
analysis identified modules of genes that are significantly perturbated in patients with NAFLD.

\section{Altered metabolism in NAFLD patients}

To further evaluate the detailed metabolic changes underlying NAFLD progression, we identified reporter metabolites (Patil and Nielsen, 2005), around which the most significant transcriptional changes occur, using differential expression data from NAFLD and network topology provided by iHepatocytes 2322 (Mardinoglu et al., 2014). (Figure S2B). Such reporter metabolites can thus be used to identify the key dysregulated regions of the metabolic network. A total of 321 metabolites were significantly ( $p$-value $<0.05$ ) associated with upregulated genes in either NAFL vs control or NASH vs control (Figure 2; Figure S2C; Dataset S4), Among these, the most significant reporter metabolites associated with upregulated genes in NAFL vs control were those involved in arginine and proline metabolism, glycerophospholipid metabolism, and nucleotide metabolism. The top reporter metabolites associated with upregulated genes between NASH and control samples involved in beta-oxidation of fatty acids, cholesterol biosynthesis, and chondroitin/heparan sulphate biosynthesis. Chondroitin sulphate (CS) and heparan sulphate (HS) are the essential components of proteoglycans (PG), which have been proposed as potential biomarkers for NASH diagnosis and staging of NAFLD by integrative analysis of transcriptomic data obtained from patients with NAFLD and GEM (Mardinoglu et al., 2014). The analyses from the current investigation utterly consistent with the previous study. In addition, we observed 215 metabolites were significantly associated with downregulated genes in NAFLD, involving in folate metabolism and oxidative phosphorylation (Figure 2; Figure S2D; Dataset S4).

\section{Validation of perturbated modules in two independent NAFLD cohorts}

To validate whether modules related to significant transcriptomics and metabolic changes in patients with NAFLD can truly reflect the perturbations in a disease-specific manner, we analysed GCNs generated using liver-biopsy proved transcriptomics datasets from two independent NAFLD cohorts with 75 and 58 samples, respectively (Azzu et al., 2021; Hoang 
et al., 2019) (to avoid repeated IDs, we assigned cohort 1 to the studied NAFLD cohort, and 2 and 3 for NAFLD cohorts for validation in the downstream analysis). By the same method constructing GCN for the first NAFLD cohort as described in Method, we identified four and eight modules of genes in NAFLD cohort 2 and 3, respectively (Figure S3A\&B). To explore module similarity among NAFLD cohorts, we calculated the Jaccard index between each pair of modules from different NAFLD cohorts and performed hypergeometric test to evaluate the significance of the observed overlap in gene members (Figure 3A, B\&C; Dataset S1). To begin with, we tested the modules between NAFLD cohort 1 and cohort 2 . The results showed that genes in cohort1_215_M4 were only significantly overlapped (29 out of 38; Jaccard index $=$ 0.388 ; hypergeometric test $\mathrm{p}$-value $=1.66 \times 10^{-69}$, Figure $3 \mathrm{~A} \& \mathrm{D}$ ) with genes in module 3 of NAFLD cohort 2 with 75 samples (cohort2_75_M3). We next tested the module pairs in NAFLD cohort 1 and cohort 3, found cohort1_215_M4 also shared 29 genes with module 7 of NAFLD cohort 3 with 58 samples (cohort_3_58_M7) (Jaccard index $=0.434$; hypergeometric test $p$-value $=3.73 \times 10^{-71}$, Figure 3B\&D). Interestingly, the genes in cohort2_75_M3 were significantly overlapped ( 45 out of 80 ; Jaccard index $=0.425$; hypergeometric test $p$-value $=$ $1.82 \times 10^{-86}$, Figure 3C\&D) with genes in cohort3_58_M7 as well.

We subsequently assessed the module similarity and overlap between any two modules between the GTEx cohort and NAFLD cohorts to validate if those conserved modules in NAFLD cohorts have a similar expression pattern in normal liver tissue. Hierarchical clustering of the Jaccard index between module pairs showed a distinct cluster consisting of cohort1_215_M4, cohort2_75_M3, and cohort3_58_M7 was only significantly overrepresented by module 3 in GTEx cohort (GTEx_M3), which contains 1,975 genes (Figure 1B; Figure S3C). We found that more than 70\% genes in cohort1_215_M4 (27 out of 38; hypergeometric test $\mathrm{p}$-value $\left.=4.11 \times 10^{-13}\right)$, cohort2_75_M3 (57 out of 80; hypergeometric test $\mathrm{p}$-value $\left.=4.97 \times 10^{-27}\right)$, and cohort3_58_M7 (50 out of 71; hypergeometric test p-value $=$ $2.92 \times 10^{-24}$ ) were included by module GTEx_M3. (Figure 4A). 
For a systematic evaluation on biological functions related to the modules, we quantified the statistical significance of enrichment of genes with the association in disease-related gene sets obtained from DisGeNET database (https://www.disgenet.org/) (Pinero et al., 2020), liverspecific proteome in Human Protein Atlas (HPA) database (http://www.proteinatlas.org/) (Uhlen et al., 2015), and KEGG pathway gene sets. We found that genes in cohort1_215_M4, cohort2_75_M3, and cohort3_58_M7 were significantly over-represented by multiple liver disease-related gene sets, including NAFLD and steatohepatitis (Figure 3E; Dataset S5). Interestingly, these three modules were also significantly enriched in heart diseases, for instance, coronary heart disease, coronary artery disease, and coronary arteriosclerosis. We further evaluated the overlap of genes in each of three disease-associated modules with 936 liver-specific genes defined by HPA (Fagerberg et al., 2014; Uhlen et al., 2015; Yu et al., 2015). The results showed that genes in cohort1_215_M4 (10 out of 38; hypergeometric test p-value $=0.0005)$, cohort2_75_M3 (30 out of 80; hypergeometric test p-value $\left.=1.68 \times 10^{-14}\right)$, and cohort3_58_M7 (22 out of 71; hypergeometric test p-value $\left.=6.02 \times 10^{-10}\right)$ are highly enriched with liver-specific genes (Figure 3F). In addition, we observed that genes in GTEx_M3, which shows high module similarity with those three modules identified in diseases cohorts, significantly enriched in the peroxisome, branched-chain amino acids (BCAAs; valine, leucine and isoleucine) degradation, and fatty acid degradation (Figure 4B). However, steroid biosynthesis and terpenoid backbone biosynthesis were the most significantly enriched pathways in all the three modules of disease cohorts. Moreover, fatty acid biosynthesis, citrate cycle (TCA cycle), and insulin signalling pathway were only significantly enriched in the modules of disease cohort(s).

\section{Topological features of genes in NAFLD-associated modules}

The analysis of topological properties can provide important information about hub genes or other influential genes that significantly impact the dynamic of the module. To understand the interplay of genes in the module, we then obtained several key network properties using the "NetworkAnalyzer" in Cytoscape to analyse the disease-associated modules. In our workflow, 
we used degree and closeness centrality to evaluate the importance of nodes in a module. In an unredirected network, the degree of a node is the number of edges linked to this node and a node with a high degree has been considered as functionally significant (Doncheva et al., 2012). Genes with high closeness centrality are considered as the controlling point of molecular communication (Miryala and Ramaiah, 2019).

Topological analysis showed that gene FDFT1 has the highest degree in both cohort1_215_M4 (26 edges) and cohort2_75_M3 (41 edges), whereas gene $M V D$ has the highest degree in cohort3_58_M7 (Figure 4C-E; Figure S4; Figure S5A; Dataset S6). The other genes with high connectivity are HMGCS1, DHCR7 and ACSS2 (23 edges, respectively), and ACAT2 (22 edges) in cohort1_215_M4; MVD (40 edges), MSMO1 and SQLE (37 edges, respectively), and IDI1 and NSDHL (36 edges, respectively) in cohort2_75_M3; IDI1(30 edges), LSS, CYP51A1, FDFT1 and SQLE (29 edges, respectively) in cohort3_58_M7. Interestingly, we observed a highly positive correlation between the degree of 33 genes overlapped in cohort1_215_M4 and cohort2_75_M3 (Spearman's correlation $=0.58, \mathrm{p}=0.00036$, Figure 4C), which indicates that those two modules have a similar topological structure. Similarly, a highly positive correlation between degree of 33 genes shared by cohort1_215_M4 and cohort3_58_M7 (Spearman's correlation $=0.73 ; p=1.7 \times 10^{-6}$, Figure 4D) and that of degree of 45 genes shared by cohort2_75_M3 and cohort3_58_M7 (Spearman's correlation $=0.81 ; \mathrm{p}=1.7 \times 10^{-11}$, Figure 4E) were also observed. Moreover, the top five genes with the highest closeness centrality in cohort1_215_M4, cohort2_75_M3 and cohort3_58_M7 are also highly conserved (Figure $\mathrm{S} 5 \mathrm{~B})$. We also observed a strong correlation between closeness centrality of shared genes in any disease-associated module pairs of NAFLD cohorts (Figure S5C).

\section{Validation of topological features in an HCC cohort}

Given that NAFLD has emerged as the fastest-growing cause of HCC (Huang et al., 2021; Ray, 2018). We next investigated whether the expression of the genes in NAFLD disease-associated modules, especially genes with high-connectivity, is predictive of patients with $\mathrm{HCC}$ using the 
https://portal.gdc.cancer.gov/projects/TCGA-LIHC) (Figure S6A). The results showed that the expression of 19 genes in cohort1_215_M4, 39 genes in cohort2_75_M3 and 40 genes in cohort3_58_M7 are significantly (log-rank p-value $<0.05)$ associated with the survival of patients, respectively (Figure S6B; Dataset S7). Among these, the high expression of 19 genes in cohort1_215_M4, 28 genes in cohort2_75_M3, and 31 genes in cohort3_58_M7 are significantly associated with an unfavourable survival of patients. For example, the high expression of FDFT1 (log-rank p-value $=6.54 \times 10^{-4}$ ) with the highest connectivity in both cohort1_215_M4 and cohort2_75_M3 and MVD $\left(\log\right.$-rank p-value $\left.=1.26 \times 10^{-3}\right)$ with the highest connectivity in cohort3_58_M7 are significantly associated with poor patient outcome (Figure S6C\&D). In addition, some of these genes have already been described as associated with NAFLD associated HCC (NAFLD-HCC). For instance, the high expression of SQLE, a second rate-limiting enzyme involved in de novo cholesterol synthesis with relatively high connectivity in disease-associated modules (Figure S4; Figure S5A), was predictive of unfavourable survival of HCC patients (log-rank p-value $=7.39 \times 10^{-4}$; Figure S7). Indeed, recent studies have demonstrated that $S Q L E$ acts as an independent prognostic factor in patients with HCC associated with NAFLD, and SQLE inhibition suppressed NAFLD-HCC growth in vitro and in vivo (Liu et al., 2018a; Ray, 2018).

\section{Identification of transcription factors that regulate the NAFLD-associated modules}

To investigate the transcriptional regulation in maintaining homeostasis and alterations in the disease state, we performed TF enrichment analysis (hypergeometric test) using the genes from the disease-associated modules and module 3 in GTEx, which shows high similarity to diseaseassociated modules (Figure 5; Dataset S8), based on TRRUST database (Han et al., 2018). Our results indicated that HNF4A, HNF1A, PPARGC1A, SREBF2 and PPARA are the most significantly enriched TFs in GTEx_M3 (Figure 5A). Meanwhile, we observed that HNF4A, SREBF1, SREBF2, YY1 and KLF13 are the significantly enriched TFs in all three diseaseassociated modules. We also found the significant upregulation of hepatic expression of 
SREBF1, SREBF2, HNF4A, and KLF13 in NAFL and NASH compared to the control group (adjusted p-value $<0.05$, Figure 5B-F; Dataset S2).

We then constructed the regulatory networks for the enriched TFs and associated targets in each of the module (Figure 5G-I; Figure S8). We observed that HNF4A, an important transcriptional factor mainly expressed in the liver, regulates the expression of genes involved in lipid metabolism and fatty acid oxidation, including cholesterol/triglyceride transporter (e.g., $A B C G 8, A B C G 5$ and MTTP), oxidoreductase (e.g., AKR1C4, CYP2D6 and CYP2B6) in the regulatory network of GTEx_M3 (Figure S8). As known, SREBF1 and SREBF2 regulate the expression of genes associated with de novo lipogenesis (DNL) (e.g., $F A S N, S C D, A C A C B$ ), synthesis and cellular uptake of cholesterol (e.g., HMGCR, FDFT1, NPC1L), respectively. Moreover, PPARA regulates the expression of genes involved in peroxisomal and mitochondrial $\beta$-oxidation, including $A C S L 1, C P T 1 A, C Y P 1 A 1$, and $A C O X 1$. Apolipoprotein $\mathrm{C} 3$ (APOC3), a central regulator of plasma triglyceride levels by inhibiting the removal of remnants of triglyceride-rich lipoproteins, is the most highly regulated gene by $H N F 4 A, N R O B 2$, PPARA and PPARGC1A in the regulatory network of GTEx_M3. Interestingly, low-density lipoprotein receptor ( $L D L R$, a key receptor that is internalized by endocytosis) is the most highly regulated genes in the disease-associated modules (Figure 5G-I) by SREBF1, SREBF2, HNF4A, YY1 and KLF13.This indicates that highly co-expressed genes involved in cholesterol metabolism in disease-associated modules are key compared to the other endocytosis-related genes that co-expressed in other modules in the same cohort. In addition to the well-established regulation of $L D L R$ activation by transcription factor SREBFs and $H N F 4 A, Y Y 1$ and $K L F 13$, two specific TFs regulating the disease-associated modules, also showed a regulatory role in the transcriptional regulation $L D L R$. Taken together, the complicated regulation of $L D L R$ in the disease-associated modules rather than endocytosis in normal liver tissue might play an essential role in the dysregulation of lipid metabolism underlying the NAFLD pathogenesis.

\section{Validation of transcription factors in a mouse NAFLD model}

Next, we generated liver transcriptomics data from a mouse NAFLD model fed by HSD and 
performed reporter TF analysis (Huang et al., 2017; Liu et al., 2019; Oliveira et al., 2008) by integrating with the same network of TF-target from TRRUST database (Han et al., 2018). We validated the TFs that are enriched in disease-associated modules (Figure 6A). The reporter TF algorithm was used to calculate the statically significant expression changes of gene sets controlled by TFs. To study the regulation of module of genes using this method, we first examined the scored reporter TFs that are significantly associated with the upregulated and downregulated genes in NAFL vs control and NASH vs control, respectively (Dataset S9). The analysis identified 12 reporter TFs of genes in cohort1_215_M4, of which 9 were associated with upregulated genes in NAFLD, including, ATF4, DDIT3, HDAC3, HNF4A, KLF5, NFYC, $S R E B F 1, S R E B F 2$, and $Y Y 1.2$ reporter TFs (VDR and WT1) are associated explicitly with downregulated genes in cohort_215_M4 between patients with NAFL and control samples. KLF13 was significantly associated with upregulated genes between NASH and control samples. Among these reporter TFs, five of them (HNF4A, SREBF1, SREBF2, YY1, and KLF13) were also identified by hypergeometric test for cohort1_215_M4 (Figure 5A). Between mice fed by HSD and chow diet (Figure 6A; Dataset S10), 5 reporter TFs (including KLF5, KLF13, SREBF1, SREBF2, and CREB1) were identified, showing significant association with upregulation of genes using corresponding human orthologs. Taken together, our analysis validated $S R E B F 1, S R E B F 2$, and $K L F 13$ as TFs that regulate the hepatic expression of genes in cohort1_215_M4.

\section{Hepatic co-expression networks reflect dysregulated cholesterol homeostasis and de novo}

\section{lipogenesis in the NAFLD cohorts}

In our network analysis, we found conserved disease-associated modules across three independent NAFLD cohorts, and more than $70 \%$ of the genes involved in the modules are associated with metabolic functions. Most of the metabolic genes in this consensus module are associated with cholesterol metabolism. For instance, 13 genes, namely HMGCS1, HMGCR, MVD, IDI1, FDPS, FDFT1, SQLE, LSS, CYP51A1, TM7SF2, NSDHL, HSD17B7, EBP and DHCR7, which are shared among the three disease-associated modules from different cohorts, 
and 5 genes, namely AACS, MVK, MSMO1, DHCR24 and SC5D which are included in at least one of the disease-associated modules from different cohorts, are involved in the endogenous synthesis of cholesterol (Figure 6B). LDLR and NPC1L1, responsible for the uptake of cholesterol, are also found in the disease-associated modules from all three cohorts. In addition, several genes, namely $A C L Y, A C S S 2, A C A C A, F A S N, S C D, F A D S 1, F A D S 2$, ELOVL6, HSD16B12, GPAM, PNPLA3 and TM6SF2, which are involved in de novo lipogenesis and lipolysis, are also included in the disease-related modules in at least one of the cohorts. Finally, a few genes encoding glycolytic enzymes, such as $G C K, P G D, A L D O B, A L D O C, P K L R, M E 1$ and $P D H B$, are also found in the disease-related modules. In summary, these indicated a strong connection between the disease-associated clusters with the cholesterol metabolism, de novo lipogenesis and glycolysis in the liver and suggested their potential roles in the development of NAFLD. 


\section{DISCUSSION}

Here, we applied a systems biology approach on human liver transcriptomics data to elucidate the dysregulated biological processes involved in NAFLD and identified potential regulators via integrating with transcriptional regulatory network. Our analysis identified a highly conserved disease-associated gene module across three different NAFLD cohorts, and this module is specified in the disease networks. At the same time, we could not be found in the network generated from normal subjects (GTEx cohort). Therefore, this gene module could play a critical role in the development of NAFLD and is closely related to the mechanism of the disease. Interestingly, we found the majority of the genes $(\sim 70 \%)$ in these diseaseassociated modules identified in the NAFLD cohort are included in the big gene module 3 of the GTEx cohort, which has 1,975 genes, and this suggested that the disease-associated module and its related biological functions were co-regulated with a large gene group in normal subjects and dysregulated with the progression of NAFLD.

In addition, our analysis provided enriched TFs that regulate the disease-associated modules, which can facilitate our understanding of the regulatory mechanism of these perturbed biological processes. The results from transcription regulatory networks analysis indicated that SREBF1, SREBF2, HNF4A, YY1, and KLF13 are the most prominent regulators of gene expression in disease-associated modules, 3 of which (SREBF1, SREBF2, and KLF13) were validated using the transcriptomics data generated from a mouse NAFLD model. Notably, KLF13 is reporter TF, specific to this disease-associated module but not for the module from the normal subjects, suggesting their potential role in the development of NAFLD (Ericsson et al., 1999; Natesampillai et al., 2006). It has been shown that selective overexpression of $Y Y 1$ resulted in massive triglyceride accumulation and moderate insulin resistance in mice fed with HFD (Lu et al., 2014) and it may a promising target for fatty liver diseases (Wu et al., 2017). We also found that $L D L R$ is a central target gene regulated by the enriched TFs in this diseaseassociated module. It has been demonstrated that multiple mechanisms are involved in protecting against excessive cholesterol accumulation in the liver (Goldstein and Brown, 2009; 
Natesampillai et al., 2006). LDLR-mediated endocytosis contributes to this process by removing approximately $70 \%$ circulating cholesterol-enriched LDL and providing feedback transcriptional regulation of cholesterol synthesis through SREBFs (Goldstein and Brown, 2009).

Our systematic analyses also highlighted the significant reporter metabolites involving in CS and HS biosynthesis, glycerophospholipid metabolism, folate metabolism and oxidative phosphorylation. Such metabolites are consistent with the findings of previous studies and could be targeted for discovery of potential biomarkers in diagnosis of NAFLD. We also found that most of the genes involved in the disease-associated module are involved in metabolic pathways such as cholesterol metabolism, DNL and glycolysis.

The liver plays a central role in cholesterol homeostasis, and growing evidence has shown that excess hepatic cholesterol causing hepatic lipotoxicity is a predominant factor in the development of human NAFLD (Ioannou, 2016; Min et al., 2012). Abundant hepatic free cholesterol stimulates Kupffer cells and hepatic stellate cells (HSCs), which are key mediators of fibrosis and inflammation as well as mitochondrial dysfunction and thus reflects the severity of disease (Musso et al., 2013). Notably, the differential expression (DE) analysis pointed out significant upregulation of critical genes (adjusted p-value $<0.05$ ) in these cholesterol-related pathways which are involved in the disease-related module, including $H M G C R$ (the principal rate-limiting enzyme in mevalonate pathway), NPC1L1 (a major gene in intestinal cholesterol absorption) in NASH compared to control group (Dataset S2). We found scavenger receptor class B type I (SCARB1), which mediate the uptake of HDL cholesterol directly, significantly increased in NAFLD patients compared to the control group. This suggests that upregulation of pathways in both synthesis and absorption of cholesterol may associate with the increased hepatic cholesterol (Ioannou, 2016), as well as increased bile acids in NAFLD patients (Jiao et al., 2018).

Additionally, recent studies have shown that high dietary cholesterol in the mice model is the causative factor for the progression of steatohepatitis to fibrosis and HCC and drives NAFLD 
associated HCC (Liu et al., 2018a; Shen et al., 2020; Zhang et al., 2021). We, therefore, investigated whether the disease-associated modules are predictive of patient outcome using the liver cancer dataset. The results from Kaplan-Meier analysis showed that the high expression of $\sim 41 \%$ genes ( 12 out of 29 genes identified in all three disease-associated modules) (Figure S6\&7) are significantly associated with poor survival of patients, for example, FDFT1, $M V D, D H C R 7, S Q L E$ and $M V D$ with high connectivity in these modules. Liu et.al. have shown that targeting SQLE can efficiently inhibit the NAFLD-HCC in cellular and animal models (Liu et al., 2018a). Considering the characteristics of the co-expression mechanism among genes with similar functions, this integrative network analysis revealed detailed molecules involved in the cholesterol mechanism. It proposed more potential therapeutical targets of effective treatment for preventing NAFLD-to-HCC progression.

Moreover, in the disease-associated module, we also found genes associated with DNL. Generally, it is believed that the triglyceride accumulation in the liver of NAFLD patients is caused by elevation of both DNL and fat uptake (Donnelly et al., 2005; Perdomo et al., 2019). However, we do not find any genes related to fat uptake in these disease-associated modules. In fact, $C D 36$, the key free fatty acid transporter, is not significantly changed between NAFLD and control group (Dataset $\mathrm{S} 2$ ). The hepatic expression of $F A B P 5$, another critical transporter for fat, is significantly decreased in the patients than normal subjects. In addition, most of DNL related genes are significantly up-regulated in the NAFL and NASH patients compared to the control group. Taken together, these suggested that the DNL, rather than free fatty acid uptake, is the source of triglyceride accumulation in NAFLD patients.

Finally, we identified a few key enzymes involved in glycolysis as well as insulin signalling pathway are included in the disease-associated module. For instance, INSIG, a key player in the insulin signalling pathway is included in the disease-associated modules among all three cohorts. A recent study has reported that $I N S I G$ is a central regulator in a negative feedback loop ensuring the balance of lipid desaturation and cholesterol composition and loss of INSIG1 improves liver damage and would healing NASH progression (Azzu et al., 2021). In addition, 
$G C K$, which is a kinase specific to glucose, is involved in the module of cohort 2 , and it is significantly up-regulated in the NAFLD patients compared to the normal subjects (Dataset S2). In our previous study, we have reported that the $G C K$ up-regulation is associated elevated insulin resistance in patients and suggested an increased influx from dietary glucose (Lee et al., 2016). Moreover, we also observed that $T K F C$, which is also involved in the module and upregulated in NAFLD patients in cohort 2. It has been reported that increased dietary fructose uptake could cause NAFLD in both mouse and human patients (Jensen et al., 2018; Loomba et al., 2021). Therefore, these results highlighted the associated between NAFLD and insulin resistance, and suggested the potentially important contribution of dietary glucose, fructose as well as sucrose to development of the disease. 


\section{METHODS AND MATERIALS}

\section{Data retrieving and pre-processing}

Each dataset was pre-processed independently:

NAFLD cohorts. hepatic RNA-seq (raw fastq files) of NAFLD cohort 1 (Govaere et al., 2020) and cohort 2 (Hoang et al., 2019) were retrieved from European Nucleotide Archive (ENA) database (https://www.ebi.ac.uk/ena/) (Amid et al., 2020) under accession numbers SRP217231 (215 biopsy-proven NAFLD patients) and SRP197353 (78 biopsy-proven NAFLD patients), respectively; Hepatic RNA-seq of NAFLD cohort 3 (Azzu et al., 2021) with 58 biopsy-proven NAFLD patients were retrieved from the ArrayExpress data repository (Parkinson et al., 2005) under accession number E-MTAB-9815. Principle component analysis (PCA) was used to detect outlier samples (Figure S1) and three outlier samples in NAFLD cohort 2 were removed based on this analysis. Afterwards, gene abundance in both transcripts per million (TPMs)) and raw count were quantified using the Kallisto (Bray et al., 2016) pipeline based on human genome (ensemble 102 version). We subsequently used DESeq2 R package following a standard protocol (Love et al., 2014) to identify differentially expressed genes (DEGs, adjusted p-value < 0.05) and performed KEGG pathway enrichment using the Platform for Integrative Analysis of Omics (PIANO) R package (Varemo et al., 2013).

GTEx cohort. The RNA-seq data with gene abundance in transcript TPMs from human tissues was retrieved from Genotype tissue expression (GTEx) (https://gtexportal.org/home/datasets) (Consortium, 2013) and remained the samples with available dataset in liver tissue.

\section{Transcriptomics data from mouse model}

Nine C57BL/6J mice were fed a standard mouse chow diet and housed in a 12-h light-dark cycle. From the age of 8 weeks, the mice were then divided into two groups of 5 mice fed with chow diet, 4 mice fed with high-sucrose diet for 3 weeks, respectively. At the age of 11 weeks, all mice are sacrificed and liver necropsy were taken for RNA sequencing. RNA sequencing library were prepared with Illumina RNA-Seq with Poly-A selections. Subsequently, the 
libraries were sequenced on NovaSeq6000 (NovaSeq Control Software 1.6.0/RNA v3.4.4) with a 2x51 setup using 'NovaSeqXp' workflow in 'S1' mode flow cell. The Bcl was converted to fastq by bc12fastq_v2.19.1.403 from CASAVA software suite (Sanger/phred33/Illumina 1.8+ quality scale). The fastq files for mice were then processed as the way that were used in human datasets mentioned above using a standard protocol of Kallisto (Bray et al., 2016).

\section{Construction of co-expression network and analysis}

Considering the dramatic increase in size owing to the many gene isoforms and non-coding RNAs (van Dam et al., 2018), we used the "protein-coding genes" for annotation of RNA-seq dataset and then constructed the co-expression network in gene level. For each dataset, we first filtered out lowly-expressed genes based on their median gene expression level (TPMs $<1)$ and constructed co-expression networks by generating gene pairs Spearman correlation ranks within liver tissue, which was performed using "spearmanr" function from SciPy (Virtanen et al., 2020) in Python 3.8. Next, we remained gene pairs with significantly (adjusted p value < 0.05) positive correlation (coefficient $>0$ ) on the network and performed module detection analysis using Leiden algorithm (Traag et al., 2019), implemented by Python package leidenalg (version 0.7.0) with "ModularityVertexPartition" to find the optimal partition. Modules with less than 30 genes were discarded to be able to get significant functional analysis results in the downstream analysis.

To explore the module similarity between different cohorts, we calculated the Jaccard index, which is simply defined as the size of the intersection between two modules divided by the size of the union of the same two modules, and used hypergeometric test to determine whether the genes in one module significantly overlapped with the genes in another module. The overlap was considered as significant when p-value less than 0.05 . Topological and node properties of modules were determined using the NetworkAnalyzer (Assenov et al., 2008) plugin implemented in Cytoscape (version, 3.8.2) (Cline et al., 2007).

\section{Functional annotation of modules in co-expression network of cohort}


KEGG enrichment analysis. we performed functional enrichment analysis for the gene lists of each module of co-expression network using hypergeometric test, which is implemented by the python package gseapy (version 0.9.16; https://github.com/zqfang/gseapy), all gene sets of KEGG pathway were obtained from database source of Enrichr (Kuleshov et al., 2016).

Disease enrichment analysis. DisGeNet (Pinero et al., 2017) is a platform integrating information of gene-disease association from several public data sources and literature. In our analysis, the lists of diseases enriched by the gene lists in each network module were retrieved from the DisGeNet database using ToppFun of the ToppGene suite (Chen et al., 2009), all gene sets in detected modules were used as background gene sets. Disease terms with BenjaminiHochberg corrected $\mathrm{p}$ value $<0.05$ were remained and top 20 for each disease-associated module were presented.

Transcription factor enrichment analysis. We retrieved the human Transcriptional Regulatory Relationship Unravelled by Sentence-based Text mining (TRRUST) v2 database (https://www.grnpedia.org/trrust/) (Han et al., 2018) and obtained the lists of transcription factor and associated targets, which derived from 7,148 PubMed articles in where small-scale experimental studies of transcriptional regulation were described. In total, 9,395 TF-target regulatory relationships of 795 TFs and 2,493 targets were supplied as database for Enrichr (Kuleshov et al., 2016), implemented by the python package gseapy (version 0.9.16; https://github.com/zqfang/gseapy).

\section{Reporter metabolite and reporter transcription factor analyses}

To investigate the detailed metabolic differences associated with NAFLD, we first performed reporter metabolites analysis (Varemo et al., 2013) using the PIANO R package with topological information from liver-specific GEM iHepatocytes2322 (Mardinoglu et al., 2014). Differential expression level of genes (log2-fold change) in each contrast and corresponding significant levels ( $\mathrm{p}$ value) were used as input.

To validate the enriched transcription factors in disease-associated modules, we also employed 
the PIANO R package to perform reporter transcription factor (TF) analysis (Huang et al., 2017; Varemo et al., 2013) in which log2-fold change and p-value of genes, as well as transcriptional regulatory information of TF-target from TRRUST database (Han et al., 2018) were used as input. Considering reporter TFs that control the expression of genes in a specific module, we assigned the p-value of genes that are not in that module as 1 in the analysis.

\section{TCGA data process and Survival analysis}

The transcript-expression level profiles (TPM) had been downloaded from Toil (Vivian et al., 2017) under the project ID of TCGA-LIHC. We screened all samples in TCGA-LIHC cohorts and kept 363 donors with both primary tumour solid tissue samples and clinical information. We only extracted tumour samples with identifier "A" for liver hepatocellular carcinoma and subsequently quantified the mRNA expression by Kallisto (Bray et al., 2016) based on the GENCODE reference transcriptome (version 23). Genes with an average TPM $>1$ were reserved for the following analysis. The clinical information was collected from TCGA PanCancer Clinical Data Resource (TCGA-CDR) (Liu et al., 2018b). Samples with a survival time of zero-day were excluded.

Genes were divided into two groups according to TPM values and examined by Kaplan-Meier survival analysis; the survival outcomes were then compared based on log-rank tests. To choose the best TPM cut-offs for grouping, all TPM values from the 20th to 80th percentiles were used to group the patients. Significant differences in the survival outcomes of the groups were examined, and the value with the lowest log-rank P-value is selected. The R package "survival" and graphics "ggplot" was used during the Kaplan-Meier analysis. Genes with logrank $\mathrm{P}$ values less than 0.05 were defined as prognostic genes. In addition, if the group of patients with high expression of a selected prognostic gene has a higher observed event than the expected event, it is an unfavourable prognostic gene; otherwise, it is a favourable prognostic gene. All analysis were applying on R. 


\section{Conflict of interest statement}

AM, JB and MU are the founder and shareholders of ScandiBio Therapeutics and they filed a patent application on the use of CMA to treat NAFLD patients. The other authors declare no conflict of interest.

\section{Acknowledgments}

AM and HY acknowledge support from the PoLiMeR Innovative Training Network (Marie Skłodowska-Curie Grant Agreement No. 812616) which has received funding from the European Union's Horizon 2020 research and innovation programme. The computations were performed on resources provided by SNIC through Uppsala Multidisciplinary Center for Advanced Computational Science (UPPMAX) under Project sllstore2017024.

\section{Funding}

This work was financially supported by ScandiBio Therapeutics and Knut and Alice Wallenberg Foundation (No. 72110). 


\section{REFERENCE}

Alonso, C., Fernandez-Ramos, D., Varela-Rey, M., Martinez-Arranz, I., Navasa, N., Van Liempd, S.M., Lavin Trueba, J.L., Mayo, R., Ilisso, C.P., de Juan, V.G., et al. (2017). Metabolomic Identification of Subtypes of Nonalcoholic Steatohepatitis. Gastroenterology 152, 1449-1461 e1447. 10.1053/j.gastro.2017.01.015.

Amid, C., Alako, B.T.F., Balavenkataraman Kadhirvelu, V., Burdett, T., Burgin, J., Fan, J., Harrison, P.W., Holt, S., Hussein, A., Ivanov, E., et al. (2020). The European Nucleotide Archive in 2019. Nucleic Acids Res 48, D70-D76. 10.1093/nar/gkz1063.

Arif, M., Zhang, C., Li, X., Gungor, C., Cakmak, B., Arslanturk, M., Tebani, A., Ozcan, B., Subas, O., Zhou, W., et al. (2021). iNetModels 2.0: an interactive visualization and database of multi-omics data. Nucleic Acids Res. 10.1093/nar/gkab254.

Asrani, S.K., Devarbhavi, H., Eaton, J., and Kamath, P.S. (2019). Burden of liver diseases in the world. J Hepatol 70, 151-171. 10.1016/j.jhep.2018.09.014.

Assenov, Y., Ramirez, F., Schelhorn, S.E., Lengauer, T., and Albrecht, M. (2008). Computing topological parameters of biological networks. Bioinformatics 24, 282-284. 10.1093/bioinformatics/btm554.

Azzu, V., Vacca, M., Kamzolas, I., Hall, Z., Leslie, J., Carobbio, S., Virtue, S., Davies, S.E., Lukasik, A., Dale, M., et al. (2021). Suppression of insulin-induced gene 1 (INSIG1) function promotes hepatic lipid remodelling and restrains NASH progression. Mol Metab 48, 101210. 10.1016/j.molmet.2021.101210.

Bray, N.L., Pimentel, H., Melsted, P., and Pachter, L. (2016). Near-optimal probabilistic RNAseq quantification. Nat Biotechnol 34, 525-527. 10.1038/nbt.3519.

Calabrese, G.M., Mesner, L.D., Stains, J.P., Tommasini, S.M., Horowitz, M.C., Rosen, C.J., and Farber, C.R. (2017). Integrating GWAS and Co-expression Network Data Identifies Bone Mineral Density Genes SPTBN1 and MARK3 and an Osteoblast Functional Module. Cell Syst 4, 46-59 e44. 10.1016/j.cels.2016.10.014.

Califano, A., Butte, A.J., Friend, S., Ideker, T., and Schadt, E. (2012). Leveraging models of cell regulation and GWAS data in integrative network-based association studies. Nat Genet 44, 841-847. 10.1038/ng.2355.

Cerami, E., Demir, E., Schultz, N., Taylor, B.S., and Sander, C. (2010). Automated network analysis identifies core pathways in glioblastoma. PLoS One 5, e8918. 10.1371/journal.pone.0008918.

Chella Krishnan, K., Kurt, Z., Barrere-Cain, R., Sabir, S., Das, A., Floyd, R., Vergnes, L., Zhao, Y., Che, N., Charugundla, S., et al. (2018). Integration of Multi-omics Data from Mouse 
Diversity Panel Highlights Mitochondrial Dysfunction in Non-alcoholic Fatty Liver Disease. Cell Syst 6, 103-115 e107. 10.1016/j.cels.2017.12.006.

Chen, J., Bardes, E.E., Aronow, B.J., and Jegga, A.G. (2009). ToppGene Suite for gene list enrichment analysis and candidate gene prioritization. Nucleic Acids Res 37, W305-311. 10.1093/nar/gkp427.

Choobdar, S., Ahsen, M.E., Crawford, J., Tomasoni, M., Fang, T., Lamparter, D., Lin, J., Hescott, B., Hu, X., Mercer, J., et al. (2019). Assessment of network module identification across complex diseases. Nat Methods 16, 843-852. 10.1038/s41592-019-0509-5.

Cline, M.S., Smoot, M., Cerami, E., Kuchinsky, A., Landys, N., Workman, C., Christmas, R., Avila-Campilo, I., Creech, M., Gross, B., et al. (2007). Integration of biological networks and gene expression data using Cytoscape. Nat Protoc 2, 2366-2382. 10.1038/nprot.2007.324.

Consortium, G.T. (2013). The Genotype-Tissue Expression (GTEx) project. Nat Genet 45, 580-585. 10.1038/ng.2653.

Doncheva, N.T., Assenov, Y., Domingues, F.S., and Albrecht, M. (2012). Topological analysis and interactive visualization of biological networks and protein structures. Nat Protoc 7, 670685. 10.1038/nprot.2012.004.

Donnelly, K.L., Smith, C.I., Schwarzenberg, S.J., Jessurun, J., Boldt, M.D., and Parks, E.J. (2005). Sources of fatty acids stored in liver and secreted via lipoproteins in patients with nonalcoholic fatty liver disease. J Clin Invest 115, 1343-1351. 10.1172/JCI23621.

El-Agroudy, N.N., Kurzbach, A., Rodionov, R.N., O'Sullivan, J., Roden, M., Birkenfeld, A.L., and Pesta, D.H. (2019). Are Lifestyle Therapies Effective for NAFLD Treatment? Trends Endocrinol Metab 30, 701-709. 10.1016/j.tem.2019.07.013.

Ericsson, J., Usheva, A., and Edwards, P.A. (1999). YY1 is a negative regulator of transcription of three sterol regulatory element-binding protein-responsive genes. J Biol Chem 274, 1450814513. 10.1074/jbc.274.20.14508.

Fagerberg, L., Hallstrom, B.M., Oksvold, P., Kampf, C., Djureinovic, D., Odeberg, J., Habuka, M., Tahmasebpoor, S., Danielsson, A., Edlund, K., et al. (2014). Analysis of the human tissuespecific expression by genome-wide integration of transcriptomics and antibody-based proteomics. Mol Cell Proteomics 13, 397-406. 10.1074/mcp.M113.035600.

Friedman, S.L., Neuschwander-Tetri, B.A., Rinella, M., and Sanyal, A.J. (2018). Mechanisms of NAFLD development and therapeutic strategies. Nat Med 24, 908-922. 10.1038/s41591018-0104-9. 
Golabi, P., Fukui, N., Paik, J., Sayiner, M., Mishra, A., and Younossi, Z.M. (2019). Mortality Risk Detected by Atherosclerotic Cardiovascular Disease Score in Patients With Nonalcoholic Fatty Liver Disease. Hepatol Commun 3, 1050-1060. 10.1002/hep4.1387.

Goldstein, J.L., and Brown, M.S. (2009). The LDL receptor. Arterioscler Thromb Vasc Biol 29, 431-438. 10.1161/ATVBAHA.108.179564.

Govaere, O., Cockell, S., Tiniakos, D., Queen, R., Younes, R., Vacca, M., Alexander, L., Ravaioli, F., Palmer, J., Petta, S., et al. (2020). Transcriptomic profiling across the nonalcoholic fatty liver disease spectrum reveals gene signatures for steatohepatitis and fibrosis. Sci Transl Med 12. 10.1126/scitranslmed.aba4448.

Han, H., Cho, J.W., Lee, S., Yun, A., Kim, H., Bae, D., Yang, S., Kim, C.Y., Lee, M., Kim, E., et al. (2018). TRRUST v2: an expanded reference database of human and mouse transcriptional regulatory interactions. Nucleic Acids Res 46, D380-D386. 10.1093/nar/gkx1013.

Hoang, S.A., Oseini, A., Feaver, R.E., Cole, B.K., Asgharpour, A., Vincent, R., Siddiqui, M., Lawson, M.J., Day, N.C., Taylor, J.M., et al. (2019). Gene Expression Predicts Histological Severity and Reveals Distinct Molecular Profiles of Nonalcoholic Fatty Liver Disease. Sci Rep 9, 12541. 10.1038/s41598-019-48746-5.

Huan, T., Zhang, B., Wang, Z., Joehanes, R., Zhu, J., Johnson, A.D., Ying, S., Munson, P.J., Raghavachari, N., Wang, R., et al. (2013). A systems biology framework identifies molecular underpinnings of coronary heart disease. Arterioscler Thromb Vasc Biol 33, 1427-1434. 10.1161/ATVBAHA.112.300112.

Huang, D.Q., El-Serag, H.B., and Loomba, R. (2021). Global epidemiology of NAFLD-related HCC: trends, predictions, risk factors and prevention. Nat Rev Gastroenterol Hepatol 18, $223-$ 238. 10.1038/s41575-020-00381-6.

Huang, M., Bao, J., Hallstrom, B.M., Petranovic, D., and Nielsen, J. (2017). Efficient protein production by yeast requires global tuning of metabolism. Nat Commun 8, 1131. 10.1038/s41467-017-00999-2.

Ioannou, G.N. (2016). The Role of Cholesterol in the Pathogenesis of NASH. Trends Endocrinol Metab 27, 84-95. 10.1016/j.tem.2015.11.008.

Ioannou, G.N., Green, P., Kerr, K.F., and Berry, K. (2019). Models estimating risk of hepatocellular carcinoma in patients with alcohol or NAFLD-related cirrhosis for risk stratification. J Hepatol 71, 523-533. 10.1016/j.jhep.2019.05.008.

Jensen, T., Abdelmalek, M.F., Sullivan, S., Nadeau, K.J., Green, M., Roncal, C., Nakagawa, T., Kuwabara, M., Sato, Y., Kang, D.H., et al. (2018). Fructose and sugar: A major mediator of non-alcoholic fatty liver disease. J Hepatol 68, 1063-1075. 10.1016/j.jhep.2018.01.019. 
Jiao, N., Baker, S.S., Chapa-Rodriguez, A., Liu, W., Nugent, C.A., Tsompana, M., Mastrandrea, L., Buck, M.J., Baker, R.D., Genco, R.J., et al. (2018). Suppressed hepatic bile acid signalling despite elevated production of primary and secondary bile acids in NAFLD. Gut 67, 1881-1891. 10.1136/gutjnl-2017-314307.

Kuleshov, M.V., Jones, M.R., Rouillard, A.D., Fernandez, N.F., Duan, Q., Wang, Z., Koplev, S., Jenkins, S.L., Jagodnik, K.M., Lachmann, A., et al. (2016). Enrichr: a comprehensive gene set enrichment analysis web server 2016 update. Nucleic Acids Res 44, W90-97. 10.1093/nar/gkw377.

Lee, S., Zhang, C., Arif, M., Liu, Z., Benfeitas, R., Bidkhori, G., Deshmukh, S., Al Shobky, M., Lovric, A., Boren, J., et al. (2018). TCSBN: a database of tissue and cancer specific biological networks. Nucleic Acids Res 46, D595-D600. 10.1093/nar/gkx994.

Lee, S., Zhang, C., Kilicarslan, M., Piening, B.D., Bjornson, E., Hallstrom, B.M., Groen, A.K., Ferrannini, E., Laakso, M., Snyder, M., et al. (2016). Integrated Network Analysis Reveals an Association between Plasma Mannose Levels and Insulin Resistance. Cell Metab 24, 172-184. 10.1016/j.cmet.2016.05.026.

Lewis, J.E., and Kemp, M.L. (2021). Integration of machine learning and genome-scale metabolic modeling identifies multi-omics biomarkers for radiation resistance. Nat Commun 12, 2700. 10.1038/s41467-021-22989-1.

Liu, D., Wong, C.C., Fu, L., Chen, H., Zhao, L., Li, C., Zhou, Y., Zhang, Y., Xu, W., Yang, Y., et al. (2018a). Squalene epoxidase drives NAFLD-induced hepatocellular carcinoma and is a pharmaceutical target. Sci Transl Med 10. 10.1126/scitranslmed.aap9840.

Liu, J., Lichtenberg, T., Hoadley, K.A., Poisson, L.M., Lazar, A.J., Cherniack, A.D., Kovatich, A.J., Benz, C.C., Levine, D.A., Lee, A.V., et al. (2018b). An Integrated TCGA Pan-Cancer Clinical Data Resource to Drive High-Quality Survival Outcome Analytics. Cell 173, 400-416 e411. 10.1016/j.cell.2018.02.052.

Liu, Z., Zhang, C., Lee, S., Kim, W., Klevstig, M., Harzandi, A.M., Sikanic, N., Arif, M., Stahlman, M., Nielsen, J., et al. (2019). Pyruvate kinase L/R is a regulator of lipid metabolism and mitochondrial function. Metab Eng 52, 263-272. 10.1016/j.ymben.2019.01.001.

Loomba, R., Friedman, S.L., and Shulman, G.I. (2021). Mechanisms and disease consequences of nonalcoholic fatty liver disease. Cell 184, 2537-2564. 10.1016/j.cell.2021.04.015.

Love, M.I., Huber, W., and Anders, S. (2014). Moderated estimation of fold change and dispersion for RNA-seq data with DESeq2. Genome Biol 15, 550. 10.1186/s13059-014-05508. 
Lu, Y., Ma, Z., Zhang, Z., Xiong, X., Wang, X., Zhang, H., Shi, G., Xia, X., Ning, G., and Li, $X$. (2014). Yin Yang 1 promotes hepatic steatosis through repression of farnesoid X receptor in obese mice. Gut 63, 170-178. 10.1136/gutjnl-2012-303150.

Mardinoglu, A., Agren, R., Kampf, C., Asplund, A., Uhlen, M., and Nielsen, J. (2014). Genome-scale metabolic modelling of hepatocytes reveals serine deficiency in patients with non-alcoholic fatty liver disease. Nat Commun 5, 3083. 10.1038/ncomms4083.

Mardinoglu, A., Boren, J., Smith, U., Uhlen, M., and Nielsen, J. (2018). Systems biology in hepatology: approaches and applications. Nat Rev Gastroenterol Hepatol 15, 365-377. 10.1038/s41575-018-0007-8.

Min, H.K., Kapoor, A., Fuchs, M., Mirshahi, F., Zhou, H., Maher, J., Kellum, J., Warnick, R., Contos, M.J., and Sanyal, A.J. (2012). Increased hepatic synthesis and dysregulation of cholesterol metabolism is associated with the severity of nonalcoholic fatty liver disease. Cell Metab 15, 665-674. 10.1016/j.cmet.2012.04.004.

Miryala, S.K., and Ramaiah, S. (2019). Exploring the multi-drug resistance in Escherichia coli O157:H7 by gene interaction network: A systems biology approach. Genomics 111, 958-965. 10.1016/j.ygeno.2018.06.002.

Mullard, A. (2020). FDA rejects NASH drug. Nat Rev Drug Discov 19, 501. 10.1038/d41573020-00126-9.

Musso, G., Gambino, R., and Cassader, M. (2013). Cholesterol metabolism and the pathogenesis of non-alcoholic steatohepatitis. Prog Lipid Res 52, 175-191. 10.1016/j.plipres.2012.11.002.

Natesampillai, S., Fernandez-Zapico, M.E., Urrutia, R., and Veldhuis, J.D. (2006). A novel functional interaction between the Sp1-like protein KLF13 and SREBP-Sp1 activation complex underlies regulation of low density lipoprotein receptor promoter function. J Biol Chem 281, 3040-3047. 10.1074/jbc.M509417200.

Nayak, R.R., Kearns, M., Spielman, R.S., and Cheung, V.G. (2009). Coexpression network based on natural variation in human gene expression reveals gene interactions and functions. Genome Res 19, 1953-1962. 10.1101/gr.097600.109.

Newsome, P.N., Buchholtz, K., Cusi, K., Linder, M., Okanoue, T., Ratziu, V., Sanyal, A.J., Sejling, A.S., Harrison, S.A., and Investigators, N.N. (2021). A Placebo-Controlled Trial of Subcutaneous Semaglutide in Nonalcoholic Steatohepatitis. N Engl J Med 384, 1113-1124. 10.1056/NEJMoa2028395. 
Oliveira, A.P., Patil, K.R., and Nielsen, J. (2008). Architecture of transcriptional regulatory circuits is knitted over the topology of bio-molecular interaction networks. BMC Syst Biol 2, 17. 10.1186/1752-0509-2-17.

Parkinson, H., Sarkans, U., Shojatalab, M., Abeygunawardena, N., Contrino, S., Coulson, R., Farne, A., Lara, G.G., Holloway, E., Kapushesky, M., et al. (2005). ArrayExpress--a public repository for microarray gene expression data at the EBI. Nucleic Acids Res 33, D553-555. 10.1093/nar/gki056.

Patil, K.R., and Nielsen, J. (2005). Uncovering transcriptional regulation of metabolism by using metabolic network topology. Proc Natl Acad Sci U S A 102, 2685-2689. 10.1073/pnas.0406811102.

Perdomo, C.M., Fruhbeck, G., and Escalada, J. (2019). Impact of Nutritional Changes on Nonalcoholic Fatty Liver Disease. Nutrients 11. 10.3390/nu11030677.

Pinero, J., Bravo, A., Queralt-Rosinach, N., Gutierrez-Sacristan, A., Deu-Pons, J., Centeno, E., Garcia-Garcia, J., Sanz, F., and Furlong, L.I. (2017). DisGeNET: a comprehensive platform integrating information on human disease-associated genes and variants. Nucleic Acids Res 45, D833-D839. 10.1093/nar/gkw943.

Pinero, J., Ramirez-Anguita, J.M., Sauch-Pitarch, J., Ronzano, F., Centeno, E., Sanz, F., and Furlong, L.I. (2020). The DisGeNET knowledge platform for disease genomics: 2019 update. Nucleic Acids Res 48, D845-D855. 10.1093/nar/gkz1021.

Ray, K. (2018). NAFLD-HCC: target cholesterol. Nat Rev Gastroenterol Hepatol 15, 390. 10.1038/s41575-018-0029-2.

Saeed, E. (2021). Core liver homeostatic co-expression networks are preserved but respond to perturbations in an organism- and disease-specific manne. Cell Syst. https://doi.org/10.1016/j.cels.2021.04.004.

Shen, T., Lu, Y., and Zhang, Q. (2020). High Squalene Epoxidase in Tumors Predicts Worse Survival in Patients With Hepatocellular Carcinoma: Integrated Bioinformatic Analysis on NAFLD and HCC. Cancer Control 27, 1073274820914663. 10.1177/1073274820914663.

Stower, H. (2021). Therapy for NASH. Nat Med 27, 21. 10.1038/s41591-020-01219-z.

Traag, V.A., Waltman, L., and van Eck, N.J. (2019). From Louvain to Leiden: guaranteeing well-connected communities. Sci Rep 9, 5233. 10.1038/s41598-019-41695-z.

Uhlen, M., Fagerberg, L., Hallstrom, B.M., Lindskog, C., Oksvold, P., Mardinoglu, A., Sivertsson, A., Kampf, C., Sjostedt, E., Asplund, A., et al. (2015). Proteomics. Tissue-based map of the human proteome. Science $347,1260419.10 .1126 /$ science.1260419. 
Uhlen, M., Zhang, C., Lee, S., Sjostedt, E., Fagerberg, L., Bidkhori, G., Benfeitas, R., Arif, M., Liu, Z., Edfors, F., et al. (2017). A pathology atlas of the human cancer transcriptome. Science 357. 10.1126/science.aan2507.

van Dam, S., Vosa, U., van der Graaf, A., Franke, L., and de Magalhaes, J.P. (2018). Gene coexpression analysis for functional classification and gene-disease predictions. Brief Bioinform 19, 575-592. 10.1093/bib/bbw139.

Varemo, L., Nielsen, J., and Nookaew, I. (2013). Enriching the gene set analysis of genomewide data by incorporating directionality of gene expression and combining statistical hypotheses and methods. Nucleic Acids Res 41, 4378-4391. 10.1093/nar/gkt111.

Virtanen, P., Gommers, R., Oliphant, T.E., Haberland, M., Reddy, T., Cournapeau, D., Burovski, E., Peterson, P., Weckesser, W., Bright, J., et al. (2020). SciPy 1.0: fundamental algorithms for scientific computing in Python. Nat Methods 17, 261-272. 10.1038/s41592-0190686-2.

Vivian, J., Rao, A.A., Nothaft, F.A., Ketchum, C., Armstrong, J., Novak, A., Pfeil, J., Narkizian, J., Deran, A.D., Musselman-Brown, A., et al. (2017). Toil enables reproducible, open source, big biomedical data analyses. Nat Biotechnol 35, 314-316. 10.1038/nbt.3772.

Wainberg, M., Kamber, R.A., Balsubramani, A., Meyers, R.M., Sinnott-Armstrong, N., Hornburg, D., Jiang, L., Chan, J., Jian, R., Gu, M., et al. (2021). A genome-wide atlas of coessential modules assigns function to uncharacterized genes. Nat Genet. 10.1038/s41588-02100840-z.

Wu, G.Y., Rui, C., Chen, J.Q., Sho, E., Zhan, S.S., Yuan, X.W., and Ding, Y.T. (2017). MicroRNA-122 Inhibits Lipid Droplet Formation and Hepatic Triglyceride Accumulation via Yin Yang 1. Cell Physiol Biochem 44, 1651-1664. 10.1159/000485765.

Ye, Q., Zou, B., Yeo, Y.H., Li, J., Huang, D.Q., Wu, Y., Yang, H., Liu, C., Kam, L.Y., Tan, X.X.E., et al. (2020). Global prevalence, incidence, and outcomes of non-obese or lean nonalcoholic fatty liver disease: a systematic review and meta-analysis. Lancet Gastroenterol Hepatol 5, 739-752. 10.1016/S2468-1253(20)30077-7.

Younossi, Z., Anstee, Q.M., Marietti, M., Hardy, T., Henry, L., Eslam, M., George, J., and Bugianesi, E. (2018). Global burden of NAFLD and NASH: trends, predictions, risk factors and prevention. Nat Rev Gastroenterol Hepatol 15, 11-20. 10.1038/nrgastro.2017.109.

Younossi, Z.M., Golabi, P., de Avila, L., Paik, J.M., Srishord, M., Fukui, N., Qiu, Y., Burns, L., Afendy, A., and Nader, F. (2019). The global epidemiology of NAFLD and NASH in patients with type 2 diabetes: A systematic review and meta-analysis. J Hepatol 71, 793-801. 10.1016/j.jhep.2019.06.021. 
Younossi, Z.M., Koenig, A.B., Abdelatif, D., Fazel, Y., Henry, L., and Wymer, M. (2016). Global epidemiology of nonalcoholic fatty liver disease-Meta-analytic assessment of prevalence, incidence, and outcomes. Hepatology 64, 73-84. 10.1002/hep.28431.

Yu, N.Y., Hallstrom, B.M., Fagerberg, L., Ponten, F., Kawaji, H., Carninci, P., Forrest, A.R., Fantom, C., Hayashizaki, Y., Uhlen, M., and Daub, C.O. (2015). Complementing tissue characterization by integrating transcriptome profiling from the Human Protein Atlas and from the FANTOM5 consortium. Nucleic Acids Res 43, 6787-6798. 10.1093/nar/gkv608.

Zhang, C., Bjornson, E., Arif, M., Tebani, A., Lovric, A., Benfeitas, R., Ozcan, M., Juszczak, K., Kim, W., Kim, J.T., et al. (2020). The acute effect of metabolic cofactor supplementation: a potential therapeutic strategy against non-alcoholic fatty liver disease. Mol Syst Biol 16, e9495. 10.15252/msb.209495.

Zhang, X., Coker, O.O., Chu, E.S., Fu, K., Lau, H.C.H., Wang, Y.X., Chan, A.W.H., Wei, H., Yang, X., Sung, J.J.Y., and Yu, J. (2021). Dietary cholesterol drives fatty liver-associated liver cancer by modulating gut microbiota and metabolites. Gut 70, 761-774. 10.1136/gutjnl-2019319664. 
A

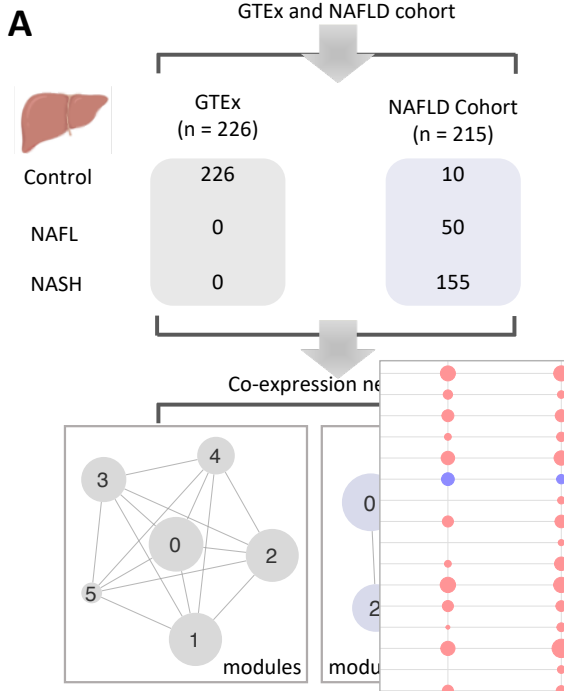

D

Number of significant DEGs

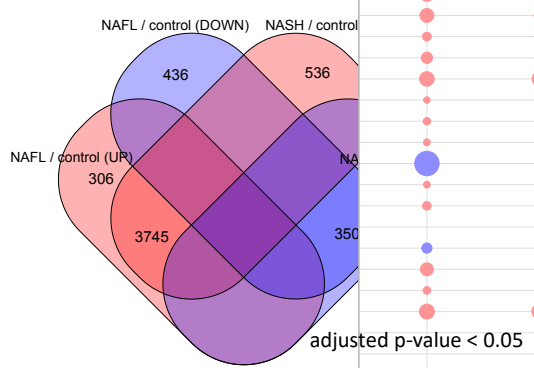

$\mathbf{F}$

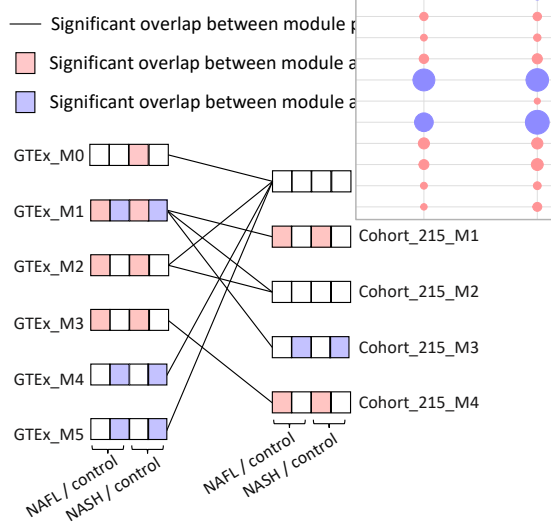

B

\begin{tabular}{lrr}
\hline Module size & GTEx & NAFLD cohort \\
\hline Module 0 & 2,732 & 2,964 \\
Module 1 & 2,614 & 2,149 \\
Module 2 & 2,595 & 2,141 \\
Module 3 & 1,975 & 332 \\
Module 4 & 1,377 & 38 \\
Module 5 & 37 & -
\end{tabular}

C

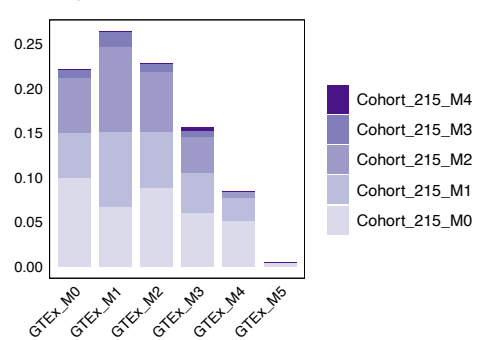

E

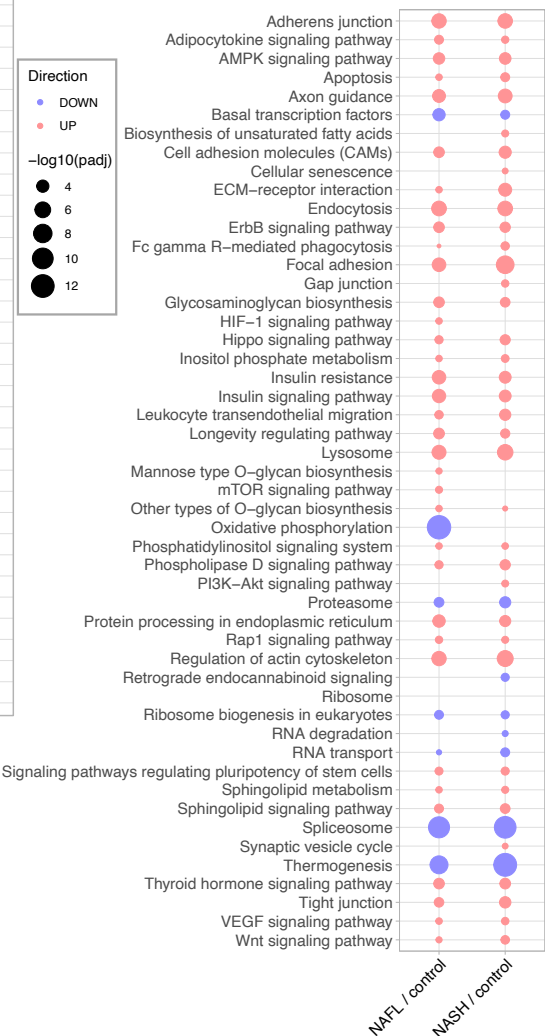

Figure 1. Sample information of studied cohorts and construction of co-expression networks. (A) Transcriptome data of liver tissue were obtained from GTEx, NAFLD cohort with 226 and 215 samples ranging from normal, NAFL, NASH, respectively. Spearman rank-order correlation coefficient analysis was applied to calculate the correlation between gene pairs after removing the lowly expressed genes (TPMs $<1$ ), and the Leiden algorithm was used to detect modules of significantly correlated genes. The label (number) of the module assigned by the algorithm. (B) The numbers of genes consist of the individual module in each cohort. (C) The bar plot shows the proportions of genes in each module identified in the NAFLD cohort compared to each module specified in the GTEx cohort. (D) The Venn diagram of differentially expressed genes (adjusted p-value $<0.05$ ) between patients with NAFLD and control samples. (E) KEGG pathway analysis showing pathways that were significantly altered in patients with NAFLD. Up-regulated and downregulated pathways are shown in blue and red, respectivelyonly pathways with adjusted p-value (padj) $<0.01$ are presented (see also Dataset S3. The size of the bubble is scaled by $-\log _{10}$ (padj) for each KEGG pathway term. (F) Significant $(p<0.05$, hypergeometric test) overlap between module pairs between GTEx and NAFLD cohorts and overlap between the module and dysregulated genes associated with NAFLD. GTEx, genotype-tissue expression; NAFLD, non-alcohol fatty acid disease; NAFL, non-alcohol fatty liver; NASH, non-alcohol steatohepatitis; TPMs, transcripts per kilobase million. 
bioRxiv preprint doi: https://doi.org/10.1101/2021.07.24.453650; this version posted July 26, 2021. The copyright holder for this preprint (which was not certified by peer review) is the author/funder, who has granted bioRxiv a license to display the preprint in perpetuity. It is made available under aCC-BY-NC-ND 4.0 International license.

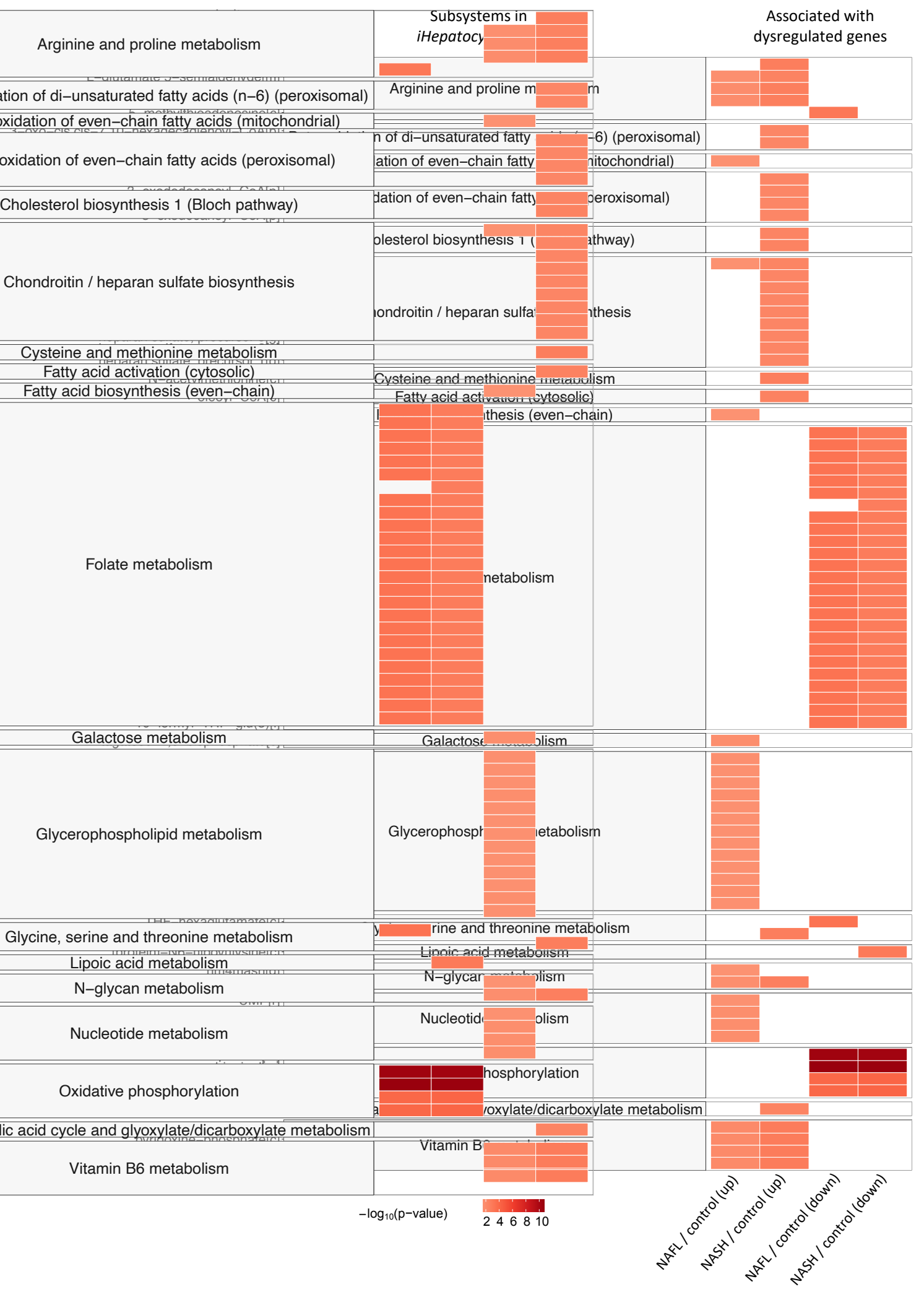

Figure 2. The most significant reporter metabolites between patients with NAFLD and control samples through the employment of iHepatocytes 2322. Reporter metabolites were calculated for the upregulated and downregulated genes for each comparison. Top30-ranked reporter metabolites and subsystems in iHepatocytes 2322 associated with up-regulated and down-regulated genes in each comparison are presented, respectively. Colour is proportional to the minus logarithm of the p-value $\left(-\log _{10}(p\right.$-value $\left.)\right)$, see also Dataset S4. 
bioRxiv preprint doi: https://doi.org/10.1101/2021.07.24.453650; this version posted July 26, 2021. The copyright holder for this preprint (which was not certified by peer review) is the author/funder, who has granted bioRxiv a license to display the preprint in perpetuity. It is made available under aCC-BY-NC-ND 4.0 International license.

A

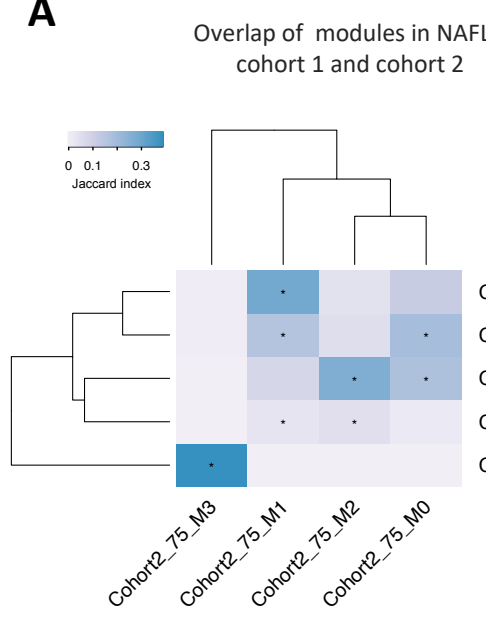

C

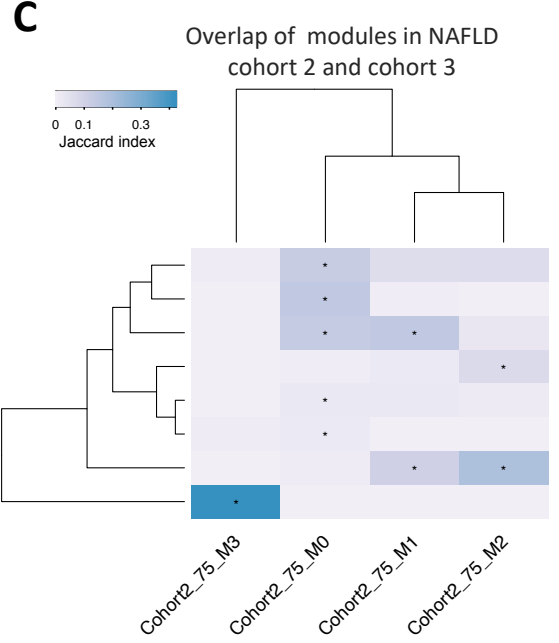

D

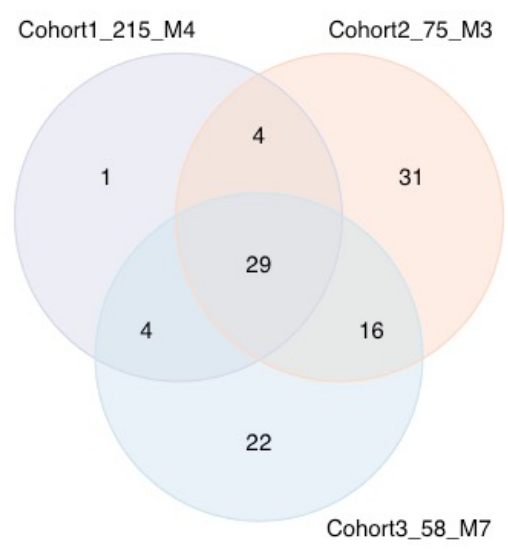

B

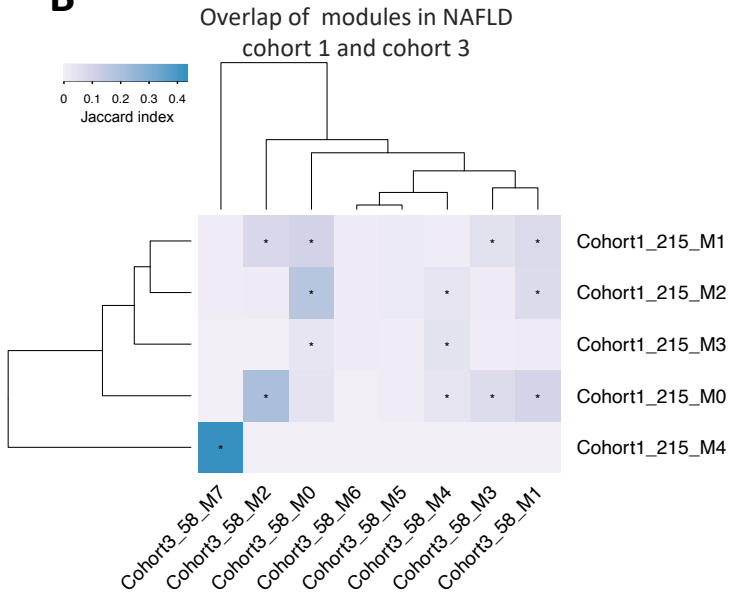

E

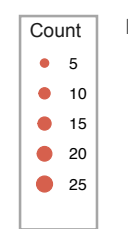

Non-alcoholic Fatty Liver Disease Coronary heart disease
Coronary Artery Disease Steatohepatitis Coronary Arteriosclerosis Liver neoplasms Cardiovascular Diseases Metabolic Diseases. Metabolic Syndrome X. Hypercholesterolemia Fatty Liver Disease

Cohort3_58_M2 Cohort3_58_M3 Cohort3_58_M0 Cohort3_58_M4 Cohort3_58_M5 Cohort3_58_M6 Cohort3_58_M1 Cohort3_58_M7

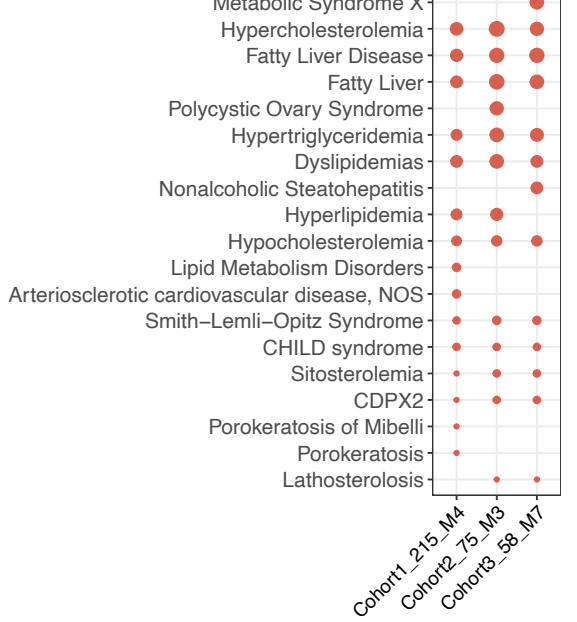

$\mathbf{F}$

Hypergeometric test between liver-specific proteome and disease-associated modules Source : HPA

\begin{tabular}{ll}
\hline Module & $p$ value \\
\hline Cohort1_215_M4 & 0.0005 \\
Cohort2_75_M3 & $1.68 \mathrm{e}-14$ \\
Cohort3_58_M7 & $6.02 \mathrm{e}-10$
\end{tabular}

Figure 3. Validation of disease-related modules using two independent NAFLD cohorts. (A-C) Hierarchical clustering of Jaccard Index between module pairs from NAFLD cohort 1 and 2; NAFLD cohort 1 and 3; NAFLD cohort 2 and 3. Color scales representing the range of the Jaccard index. asterisk indicates the statistical significance of the overlap between gene members in any two modules from the different cohorts. (D) Venn diagram shows numbers of genes overlapped between cohort1_215_M4, cohort2_75_M3, and cohort3_58_M7. (E) Dot-plot heatmap shows top 20 significantly ('q-value FDR B\& $\bar{H}$ ' $<0.05$ ) enriched diseases by genes in each module (cohort1_215_M4, cohort2_75_M3, and cohort3_58_M7). The size of each dot is proportional to the number of genes enriched in each disease term. (F) The table shows the results from a hypergeometric test between liver-specific proteome (HPA) and disease-associated modules in NAFLD cohorts, p-value less than 0.05. 
bioRxiv preprint doi: https://doi.org/10.1101/2021.07.24.453650; this version posted July 26, 2021. The copyright holder for this preprint (which was not certified by peer review) is the author/funder, who has granted bioRxiv a license to display the preprint in perpetuity. It is made available under aCC-BY-NC-ND 4.0 International license.

A

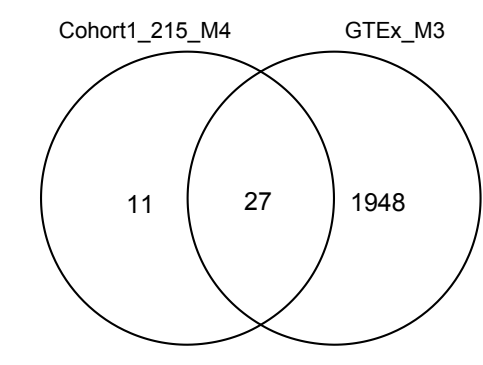

B

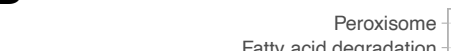

Metabolism of xenobiotics Fatty acid degradation Retinol metabolism Tryptophan metabolism Glycine, serine and threonine metabolism Primary bile acid biosynthesis Ascorbate and aldarate metabolism. Steroid biosynthesis -Alanine metabolism Tyrosine metabolism Pentose and glucuronate interconversions Alanine, aspartate and glutamate metabolism Histidine metabolism Terpenoid backbone biosynthesis Pyruvate metabolism Porphyrin and chlorophyll metabolism Arginine biosynthesis Arginine and proline metabolism proline metabolism
ABC transporters Cysteine and methionine metabolism Glutathione metabolism Glutathione metabolism Glycolysis / Gluconeogenesis
Biosynthesis of unsaturated fatty acids Propanoate metabolism Valine, leucine and isoleucine degradation PPAR signaling pathway One carbon pool by folateSynthesis and degradation of ketone bodies Complement and coagulation cascades Phenylalanine metabolism Fatty acid biosynthesis Steroid hormone biosynthesis Cholesterol metabolism Glycerolipid metabolism Glycerolipid metabolism Butanoate metabolism Glyoxylate and dicarboxylate metabolism
AMPK signaling pathway
Vitamin digestion and absorption Glyoxylate and dicarboxylate metabolism
AMPK signaling pathway
Vitamin digestion and absorption Pantothenate and CoA biosynthesis
Citrate cycle (TCA cycle)

Nicotinate and nicotinamide metabolism Insulin signaling pathway Fructose and mannose metabolism Fatty acid elongation Galactose metabolism Arachidonic acid metabolism Arachidonic acid metabolism
Glucagon signaling pathway Glucagon signaling pathway
Fat digestion and absorption Fat digestion and absorption Pentose phosphate pathway Proximal tubule bicarbonate reclamation -
Linoleic acid metabolism Amino sugar and nucleotide sugar metabolism -
Starch and sucrose metabolismPhenylalanine, tyrosine and tryptophan biosynthesis Pyrimidine metabolism
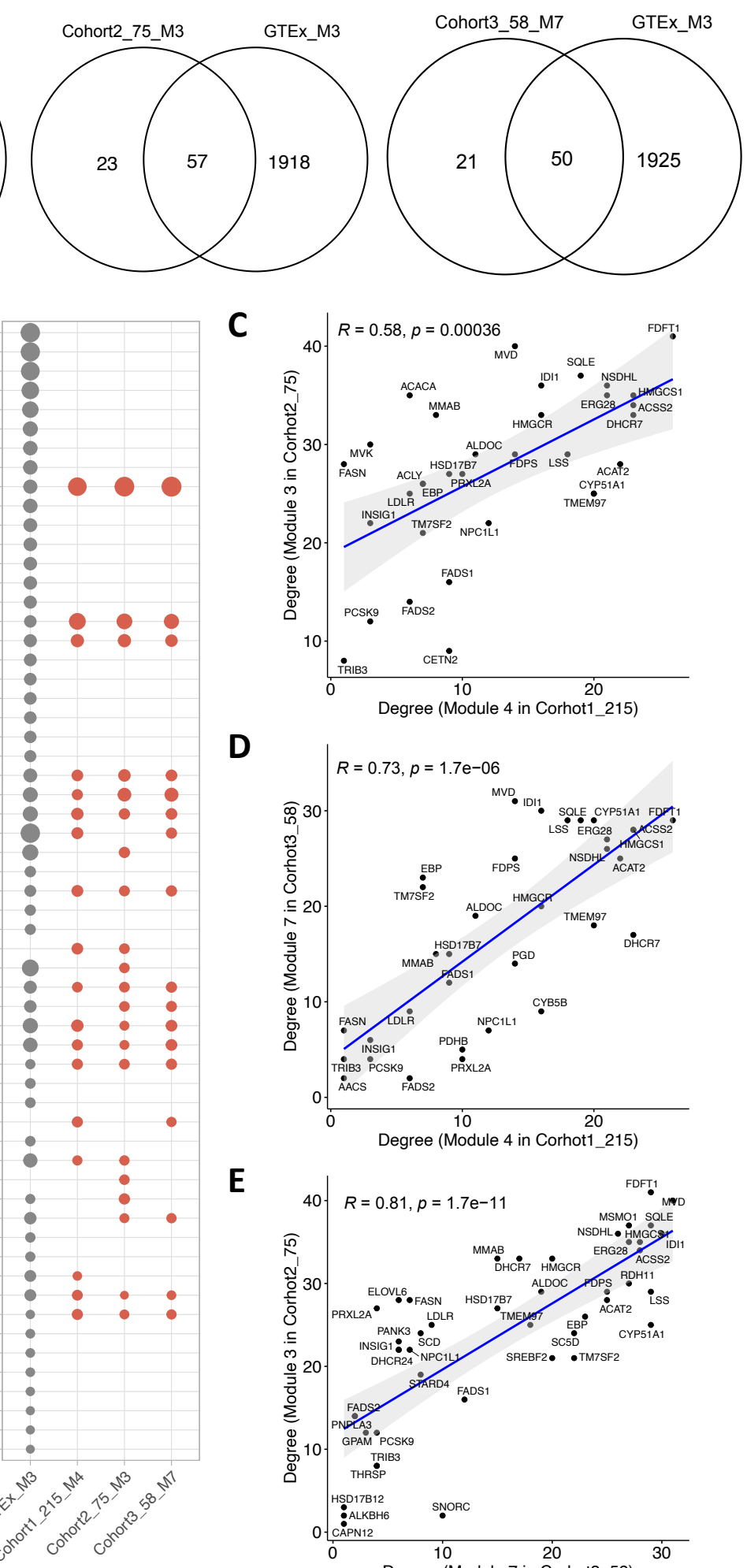

C

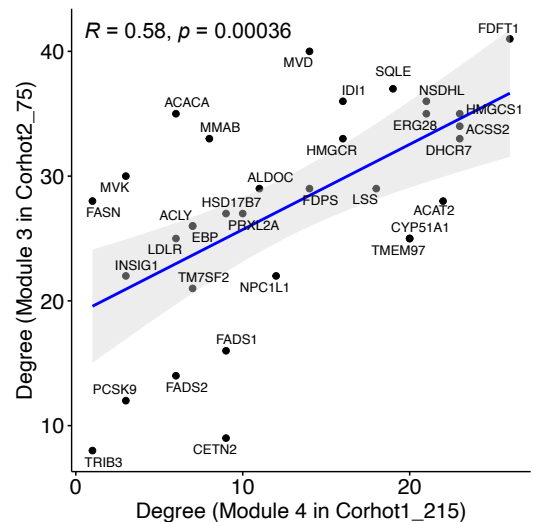

D

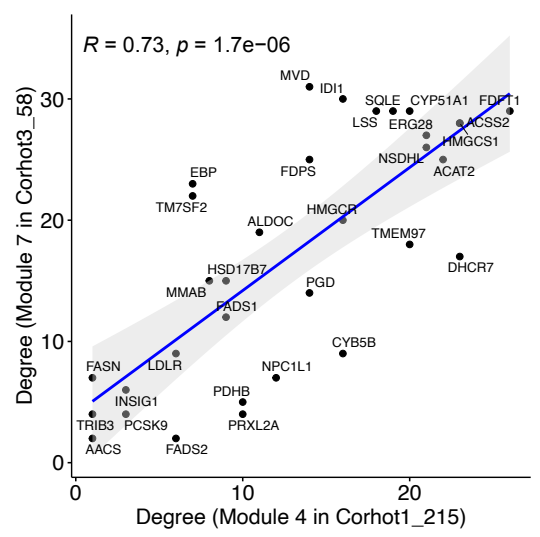

E

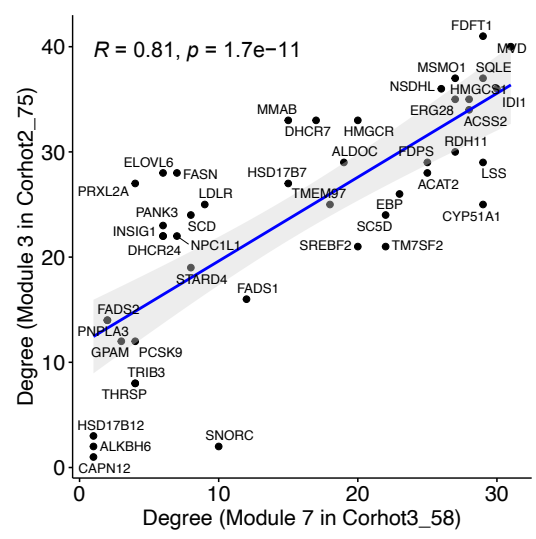

Figure 4. (A) Venn diagram shows numbers of genes overlapped between GTEx_M3 cohort and cohort1_215_M4, cohort2_75_M3, and cohort3_58_M7, respectively. (B) Dot-plot heatmaps are showing KEGG pathways enriched in different modules. The colour differences of dots indicate the studied cohort (GTEx or NAFLD) in which the module detected. The size of each dot is proportional to the significance (- $\log _{10}$ (padj); padj represents ' $q$-value FDR B\&H' with value $<0.05$ ) of enrichment for each KEGG pathway term. (C-E) Correlation between degree among disease-associated modules from different cohorts. The correlation was evaluated by Spearman correlation coefficients. 
A

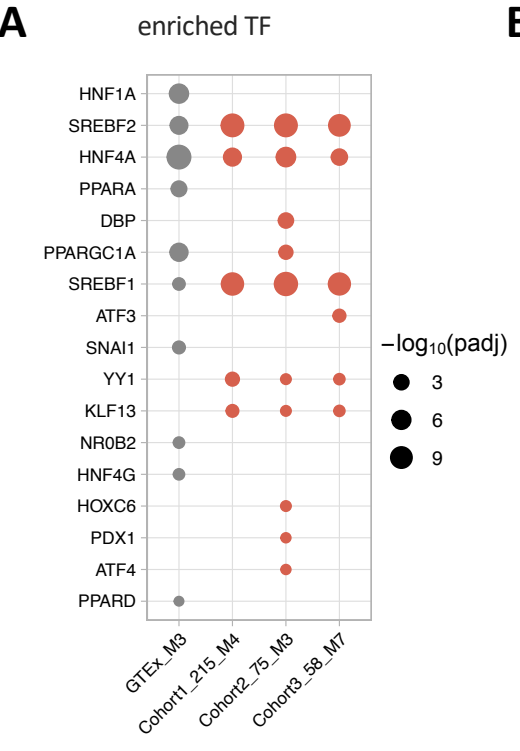

$\mathbf{F}$

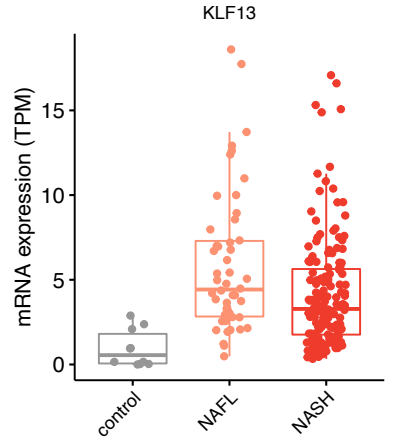

B

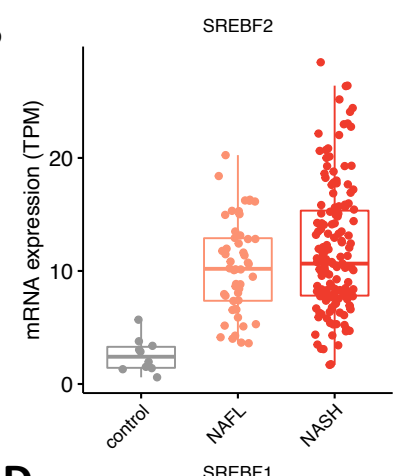

D

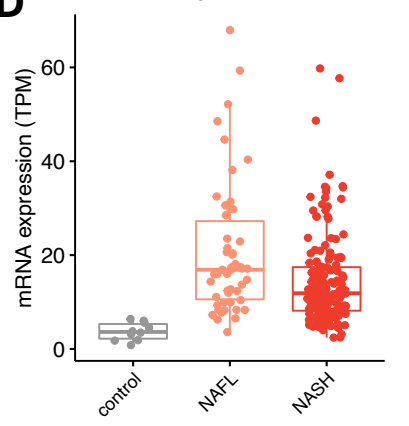

C

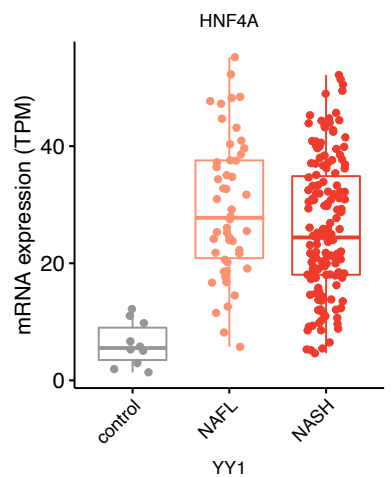

$\mathbf{E}$

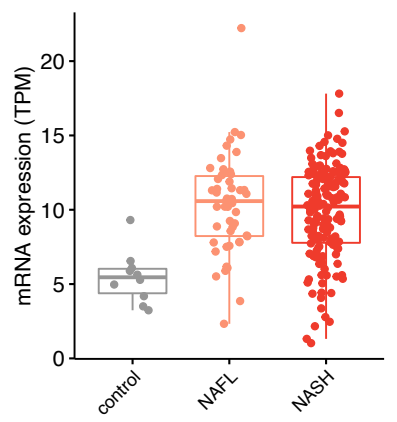

H

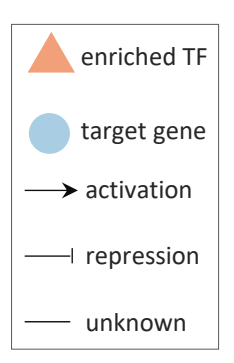

\section{G}
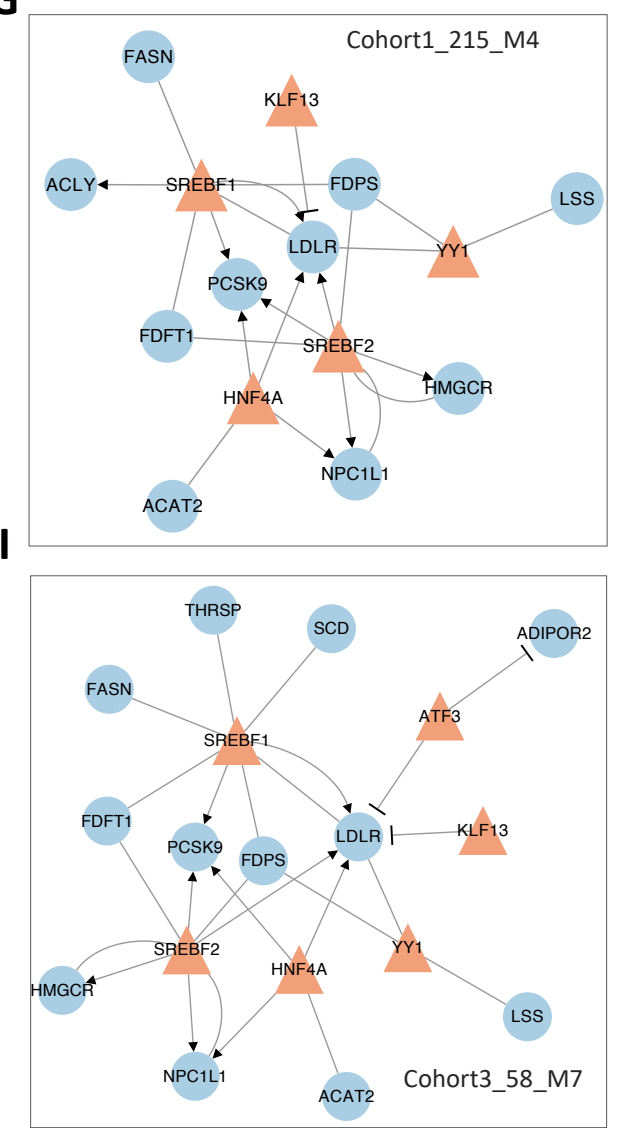

Figure 5. Regulatory relationship between an enriched transcription factor and associated target genes in diseaseassociated modules. (A) enriched transcription factors in GTEx_M3, cohort1_215_M4, cohort2_75_M3, and cohort3_58_M7. (B-F) mRNA hepatic expression of the enriched transcription factors in disease-associated modules, including SREBF2, HNF4A, SREBF1, YY1, and KLF13. (G-I) the regulatory network between enriched transcription factors and associated target genes in cohort1_215_M4, cohort2_75_M3, and cohort3 58_M7. The regulation between transcription factor and its target was retrieved from the TRRUST database. 
bioRxiv preprint doi: https://doi.org/10.1101/2021.07.24.453650; this version posted July $26,2021$. The copyright holder for this preprint (which was not certified by peer review) is the author/funder, who has granted bioRxiv a license to display the preprint in perpetuity. It is made available under aCC-BY-NC-ND 4.0 International license.

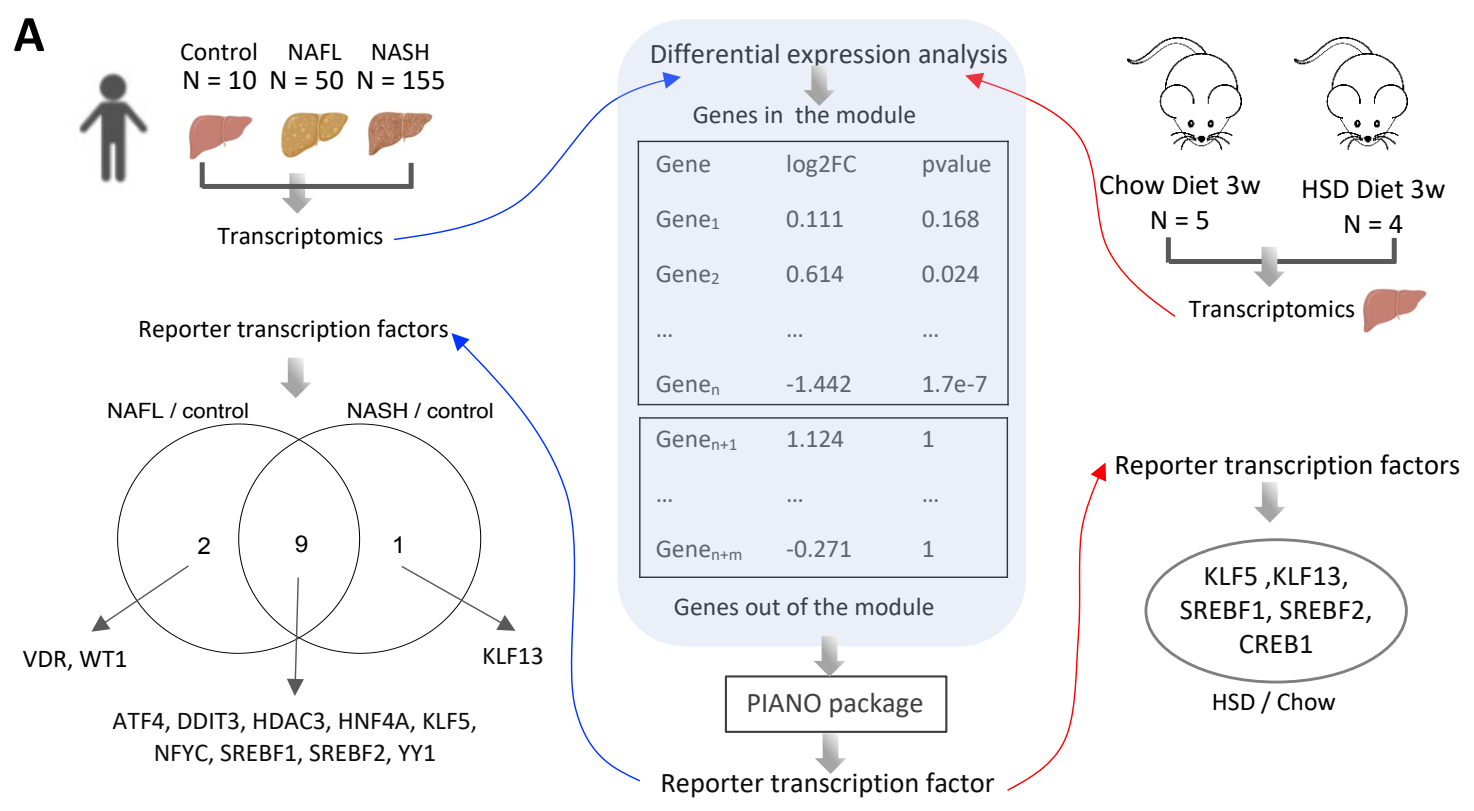

B

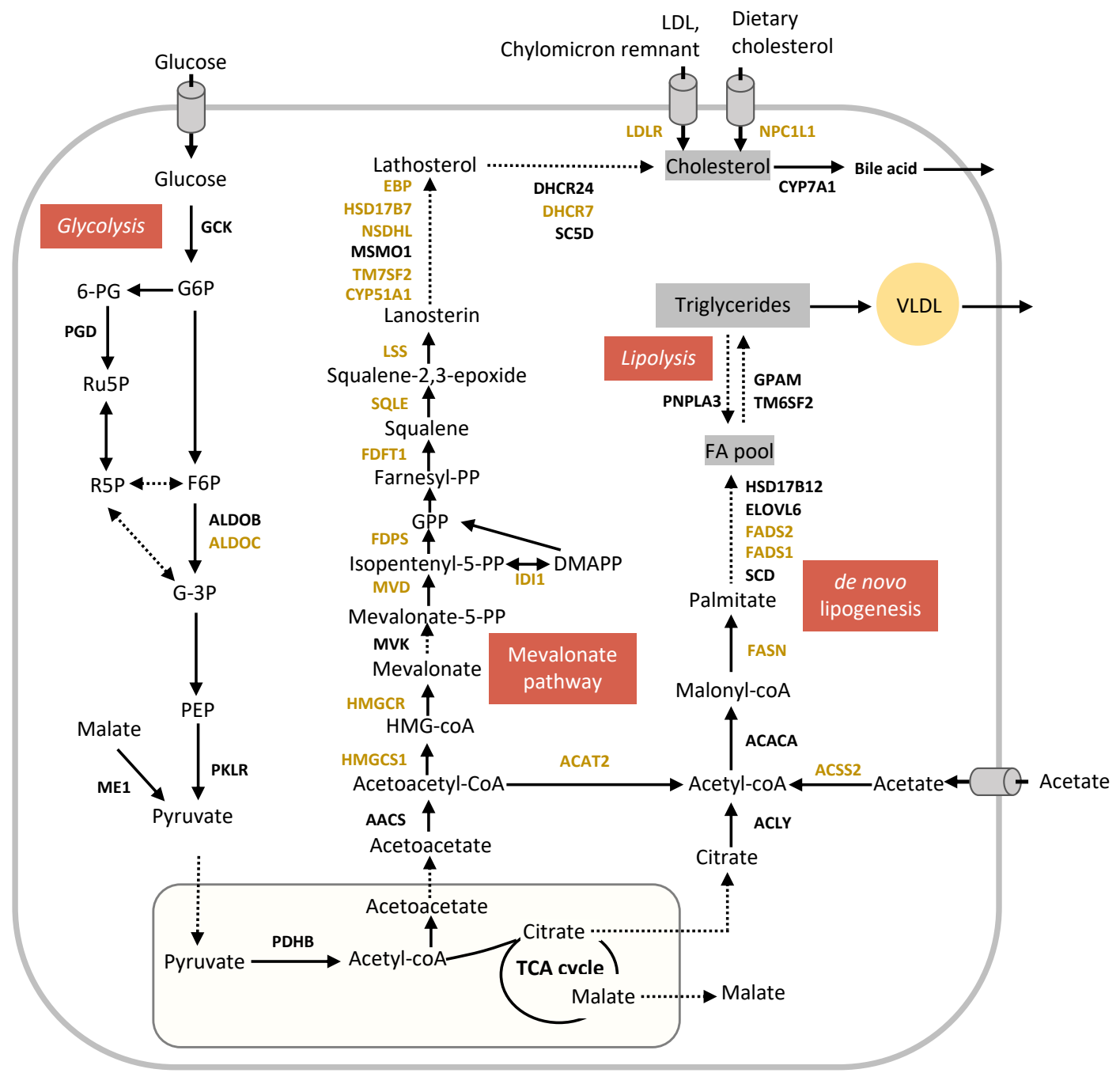

Figure 6. (A) Reporter TF analysis was used to validate TFs identified in disease-associated modules using transcriptomics data of NAFLD cohort 1 and newly generated from a mouse NAFLD model. (B) Conserved disease-associated modules revealed the dysregulation in the mevalonate pathway, de novo lipogenesis, glycolysis, and lipolysis. 